\title{
Imaging and serum biomarkers reflecting the functional efficacy of extended erythropoietin treatment in rats following infantile traumatic brain injury
}

\author{
Shenandoah Robinson, MD, ${ }^{1-3,7}$ Jesse L. Winer, MD,,2 Justin Berkner, BA, ${ }^{1,4}$ \\ Lindsay A. S. Chan, BA, ${ }^{1,2}$ Jesse L. Denson, PhD, ${ }^{8}$ Jessie R. Maxwell, MD, ${ }^{8}$ Yirong Yang, PhD, ${ }^{10}$ \\ Laurel O. Sillerud, PhD, ${ }^{10}$ Robert C. Tasker, MBBS, MD, ${ }^{1,3,6}$ William P. Meehan III, MD, ${ }^{1,5}$ \\ Rebekah Mannix, MD, MPH, ${ }^{1,4}$ and Lauren L. Jantzie, $\mathrm{PhD}^{8,9}$ \\ ${ }^{1}$ Brain Injury Center and Departments of ${ }^{2}$ Neurosurgery, ${ }^{3}$ Neurology, ${ }^{4}$ Emergency Medicine, ${ }^{5}$ Sports Medicine, and \\ ${ }^{6}$ Anesthesiology, ${ }^{7}$ F. M. Kirby Center for Neurobiology, Boston Children's Hospital, Harvard Medical School, Boston, \\ Massachusetts; and Departments of ${ }^{8}$ Pediatrics, ${ }^{9}$ Neurosciences, and ${ }^{10}$ Neurology, University of New Mexico School of Medicine, \\ Albuquerque, New Mexico
}

OBJECTIVE Traumatic brain injury (TBI) is a leading cause of death and severe morbidity for otherwise healthy fullterm infants around the world. Currently, the primary treatment for infant TBI is supportive, as no targeted therapies exist to actively promote recovery. The developing infant brain, in particular, has a unique response to injury and the potential for repair, both of which vary with maturation. Targeted interventions and objective measures of therapeutic efficacy are needed in this special population. The authors hypothesized that MRI and serum biomarkers can be used to quantify outcomes following infantile TBI in a preclinical rat model and that the potential efficacy of the neuro-reparative agent erythropoietin (EPO) in promoting recovery can be tested using these biomarkers as surrogates for functional outcomes.

METHODS With institutional approval, a controlled cortical impact $(\mathrm{CCl})$ was delivered to postnatal Day $(\mathrm{P}) 12$ rats of both sexes (76 rats). On postinjury Day (PID)1, the $49 \mathrm{CCl}$ rats designated for chronic studies were randomized to EPO (3000 U/kg/dose, CCI-EPO, 24 rats) or vehicle (CCl-veh, 25 rats) administered intraperitoneally on PID1-4, 6, and 8. Acute injury (PID3) was evaluated with an immunoassay of injured cortex and serum, and chronic injury (PID13-28) was evaluated with digitized gait analyses, MRI, and serum immunoassay. The CCl-veh and CCI-EPO rats were compared with shams (49 rats) primarily using 2-way ANOVA with Bonferroni post hoc correction.

RESULTS Following CCl, there was $4.8 \%$ mortality and $55 \%$ of injured rats exhibited convulsions. Of the injured rats designated for chronic analyses, $8.1 \%$ developed leptomeningeal cyst-like lesions verified with MRI and were excluded from further study. On PID3, Western blot showed that EPO receptor expression was increased in the injured cortex $(p=0.008)$. These Western blots also showed elevated ipsilateral cortex calpain degradation products for $\alpha$ ll-spectrin ( $\alpha$ ll-SDPs; $p<0.001$ ), potassium chloride cotransporter 2 (KCC2-DPs; $p=0.037$ ), and glial fibrillary acidic protein (GFAP-DPs; $p=0.002$ ), as well as serum GFAP (serum GFAP-DPs; $p=0.001$ ). In injured rats multiplex electrochemiluminescence analyses on PID3 revealed elevated serum tumor necrosis factor alpha (TNF $\alpha ; p=0.01$ ) and chemokine (CXC) ligand 1 (CXCL1). Chronically, that is, in PID13-16 CCl-veh rats, as compared with sham rats, gait deficits were demonstrated $(p=0.033)$ but then were reversed $(p=0.022)$ with EPO treatment. Diffusion tensor MRI of the ipsilateral and contralateral cortex and white matter in PID16-23 CCl-veh rats showed widespread injury and significant abnormalities of functional anisotropy (FA), mean diffusivity (MD), axial diffusivity (AD), and radial diffusivity (RD); $M D, A D$, and RD improved after EPO treatment. Chronically, P13-P28 CCI-veh rats also had elevated serum CXCL1 levels, which normalized in CCI-EPO rats.

ABBREVIATIONS $\alpha$ ll-SDPs = $\alpha$ ll-spectrin DPs; $A D=$ axial diffusivity; $C C l$ = controlled cortical impact; $C X C L 1=$ chemokine (CXC) ligand $1 ;$ DP = degradation product; $D$ TI = diffusion tensor imaging; EP = echo planar; EPO = erythropoietin; EPOR = EPO receptor; FA = fractional anisotropy; GFAP = glial fibrillary acidic protein; IFN $\gamma=$ interferon gamma; IL = interleukin; $\mathrm{KCC} 2$ = potassium chloride cotransporter $2 ; \mathrm{MD}=$ mean diffusivity; $\mathrm{MECl}=$ multielectrochemiluminescence; $\mathrm{P}=$ postnatal day; $\mathrm{PID}=$ postinjury day; $\mathrm{RD}=$ radial diffusivity; $\mathrm{ROI}=$ region of interest; $\mathrm{SWI}=$ susceptibility-weighted imaging; $\mathrm{TBI}=$ traumatic brain injury; TNF $\alpha=$ tumor necrosis factor alpha; veh = vehicle. SUBMITTED September 13, 2015. ACCEPTED October 29, 2015.

INCLUDE WHEN CITING Published online February 19, 2016; DOI: 10.3171/2015.10.PEDS15554. 
CONCLUSIONS Efficient translation of emerging neuro-reparative interventions dictates the use of age-appropriate preclinical models with human clinical trial-compatible biomarkers. In the present study, the authors showed that $\mathrm{CCl}$ produced chronic gait deficits in P12 rats that resolved with EPO treatment and that chronic imaging and serum biomarkers correlated with this improvement.

http://thejns.org/doi/abs/10.3171/2015.10.PEDS15554

KEY WORDS controlled cortical impact; diffusion tensor imaging; diffusivity; erythropoietin; infant; serum biomarker; traumatic brain injury; trauma

$\mathrm{T}$ HE global burden of traumatic brain injury (TBI) in infants is immense not only because of the lifelong deficits it causes, but also for its societal costs. In the US, TBI is the leading cause of death and severe morbidity in full-term infants who are born healthy. ${ }^{55,59,75}$ While severe infant TBI is not as well documented in many other countries, the incidence and outcomes are probably similar to, if not worse than, those in the US. Despite the global toll of infantile TBI, little progress has been made toward developing effective interventions to treat this population. Children in the US under 2 years of age most commonly suffer TBI from falls (44\%), abusive head trauma $(22 \%)$, motor vehicle or pedestrian-motor vehicle collisions $(14 \%)$, and other injuries $(7 \%)^{47,73}$ such as television tip-over. ${ }^{62,81}$ The majority of these injuries involve a component of impact. The observed variability in age-specific responses to emerging neuro-reparative interventions has emphasized the need for the development of age-appropriate preclinical models with translational potential. To address this need, we approached the present study with 2 primary objectives. Our first was to quantify clinically relevant chronic outcomes with imaging and serum biomarkers by using an age-appropriate model of infantile impact-related TBI. The second objective was to test whether the emerging neuro-reparative agent erythropoietin (EPO), administered via an extended dosing regimen based on its CNS mechanisms of repair, could alter chronic outcomes after infant TBI.

Erythropoietin has multiple mechanisms of action that can be beneficial for treating TBI, ${ }^{14,69,90}$ including enhancing the survival and maturation of oligodendrocytes and neurons after injury. ${ }^{38,53,97}$ Similar to the effect on erythroblasts in the bone marrow, unbound EPO receptors (EPORs) promote neural cell apoptosis. ${ }^{43,44}$ In the developing brain, EPOR expression disproportionately increases after injury relative to ligand, ${ }^{41,53}$ promoting neural cell death after injury. Exogenous EPO administration can restore the EPO ligand/receptor balance and thus promote neural cell survival and differentiation. ${ }^{53}$ For preterm infants with CNS injury, high doses of EPO over an extended regimen are safe ${ }^{24,40}$ and effective for neuroprotection. ${ }^{46,63}$ Accumulating evidence suggests that in order for EPO to be effective at neurorepair, the agent must be given at high dosages for an extended period. ${ }^{53,57,77,103}$ Extended EPO treatment has shown promise in adult trials of other disease processes ${ }^{60,61}$ but not in a recent adult TBI trial. ${ }^{77}$ The pronounced efficacy of EPO in the developing brain compared with the mature CNS suggests that age at the time of treatment may also be important. Indeed, the characteristics of EPO, including activity against multiple pathophysiological mechanisms known to contribute to secondary injury after TBI and a therapeutic window sufficiently long to be practical in the clinical setting, support its protective capacity after infant TBI. ${ }^{71}$

We developed a postnatal day (P)12 controlled cortical impact (CCI) injury model in Sprague-Dawley rats, in which P7-P9 is equivalent to full term in humans and P17P21 is considered toddler age in humans. ${ }^{1,87}$ Controlled cortical impact produces contusion and injury in the cortex, white matter, and hippocampus immediately beneath the impact and diffuse injury in the hemisphere contralateral to the impact. ${ }^{10,27,86}$ Unlike fluid percussion models that have been increasingly used to study sports concussion and blast-induced TBI, CCI mimics biomechanical events contributing to injury such as the force and tissue deformation common to mechanisms in infants with head injury.

We evaluated chronic microstructural abnormalities using ex vivo MR diffusion tensor imaging (DTI). Fractional anisotropy (FA) of the diffusion tensor provides a gross quantification of the spectrum of microstructural integrity on a scale from 0 to 1, with lower numbers representing less integrity due to microstructural damage. ${ }^{48,95}$ In the subacute and chronic phases, mean diffusivity (MD), axial diffusivity (AD), and radial diffusivity (RD) measure the directionality of water diffusion at the cellular and organelle level. ${ }^{7,48}$ We hypothesized that CCI at P12 could alter chronic microstructural parameters and provide an MRI biomarker of infantile TBI. While serum biomarkers of CNS injury do not localize structural damage as imaging does, serum levels of degradation products (DPs) from CNS molecules critical to neural cell function and proinflammatory cytokines may reflect the biological severity as well as the recovery of the injury. Thus, serum biomarkers may allow stratification of patients and individual tailoring of therapeutic interventions. We also expected serum biomarkers to reflect both the acute and chronic alterations produced by the P12 CCI. We found that serum glial fibrillary acidic protein (GFAP)-DPs and tumor necrosis factor alpha (TNF $\alpha$ ) levels were elevated 3 days postinjury (PID3). An unexpected finding was the elevated chemokine (CXC) ligand 1 (CXCL1) levels in serum in both the acute and chronic phases of injury. Finally, we tested the hypothesis that EPO treatment would alter functional outcomes and that these changes would be measureable with MRI and serum biomarkers. We found chronic gait deficits that resolved with EPO treatment and were accompanied by normalization of MRI abnormalities and serum CXCL1 levels. 


\section{Methods}

\section{Sample Size Estimation}

A schematic of the analyses performed in this study (125 rats total) is presented in Fig. 1. Because functional outcomes have not been defined for gait in a model of infant TBI, gait analyses from other types of perinatal brain injury were used to estimate the sample size needed to detect a significant difference. Previous computerized analyses of videotaped gait studies (Digigait, Mouse Specifics, Inc.) from the same rat strain at the same age showed that a significant change in gait was detectable with a sample size of at least 8 rats per group. ${ }^{34,35}$ A similar strategy was used to estimate the sample sizes needed to detect group differences in mRNA and protein levels from the brain for EPOR levels, ${ }^{53}$ cytokines and calpain DPs, ${ }^{37,107}$ serum cytokine changes following TBI, ${ }^{98}$ and microstructural DTI alterations on ex vivo MRI. ${ }^{36}$ A sample size of 6 per group would be expected to detect a $20 \%$ difference with a Type 1 error of 0.05 with a SD of $20 \%$ of the mean. Given the high resource intensity of these studies, this initial pilot study was not powered to detect sex differences in the degree of injury or EPO responsiveness.

\section{Infant Rat CCl}

All procedures were performed in accordance with the National Institutes of Health Guide for the Care and Use
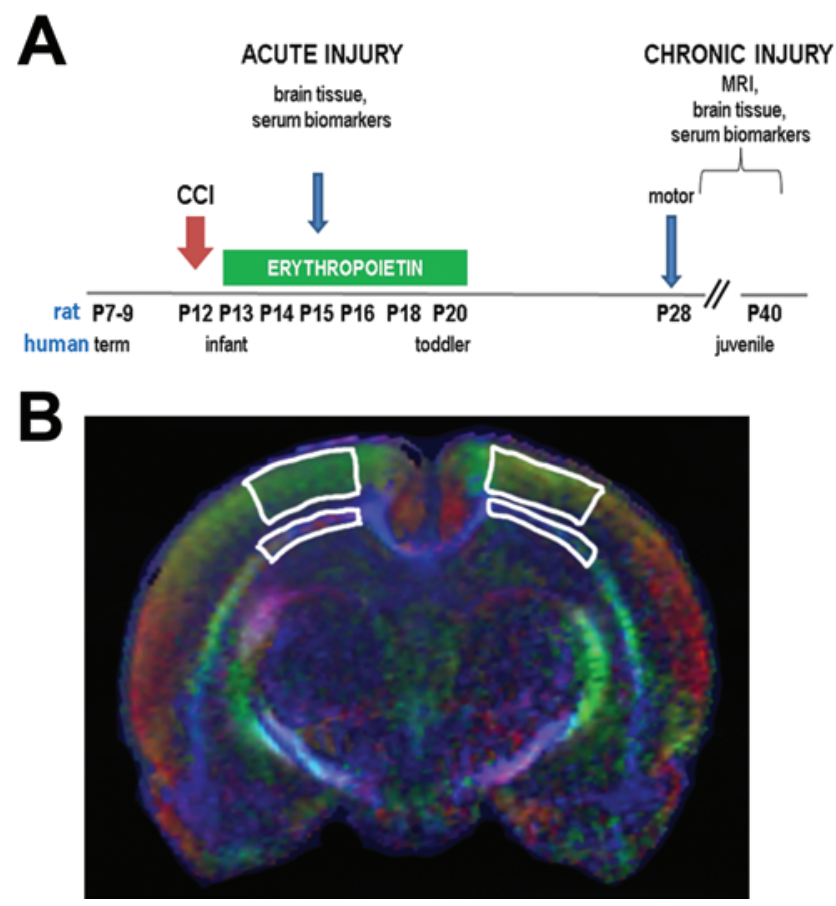

FIG. 1. A: Schematic of experimental paradigm. The P12 rat pups, approximately equal to 6 -month-old human infants, underwent $\mathrm{CCl}$. A subset of $\mathrm{P} 15$ sham and $\mathrm{CCl}$-veh rats underwent studies in the acute period at 72 hours after $\mathrm{CCl}$ (49 rats). For chronic studies (76 rats), $\mathrm{CCl}$ rats were randomized to receive $3000 \mathrm{U} / \mathrm{kg}$ of EPO or vehicle on PID1, 2, 3, 4, 6, and 8 and underwent analysis beginning at P28 (PID16). B: Regions of interest used for DTI analysis for the ipsilateral and contralateral white matter (thin strips) and cortex (wide strips). Figure is available in color online only. of Laboratory Animals and were approved by the institutional animal care and use committees at the Boston Children's Hospital and the University of New Mexico Health Sciences Center. As stated above, P7-P10 in rats is equivalent to full term in humans, P17-P21 is equivalent to a toddler, and P25-P35 is equivalent to an older child. ${ }^{87}$ We estimated that P12 is approximately equivalent to a several-month-old infant. Given our experience in producing a consistent CCI injury in mice ${ }^{49,50-52}$ and because the P12 rat pup is approximately similar in size to an adult mouse, we used a mouse CCI device to deliver a brain injury to P12 rats. Under isoflurane anesthesia, P12 Sprague-Dawley rat pups underwent a 5-mm-diameter left craniectomy. Care was taken to avoid dural injury. The rat heads were fixed with the animals prone (76 rats), and an air-powered piston (3-mm diameter, Amscien Instruments) delivered a CCI to a $0.6-\mathrm{mm}$ depth at a velocity of $6 \mathrm{~m} / \mathrm{sec}$ to the left parietal lobe consistent with our prior reports. ${ }^{49,51}$ The main point of impact in rats this age was the parietal cortex (bregma $-3.0 \mathrm{~mm}$ ). Sham animals (49 rats) underwent an equal time (15 $\mathrm{min})$ of anesthesia with a scalp incision but no craniectomy. Body temperatures were maintained throughout the brief procedure. Note that pilot studies have shown that craniectomy in sham rats resulted in mild intracranial injury and that intracranial injury has occurred from craniotomy alone in mice. ${ }^{83}$ All animals recovered with their mothers. Pups were reared with dams until P21, when they were weaned and housed in single-sex groups of 2-3 animals per cage, with lighting on from $7 \mathrm{Am}$ to $7 \mathrm{PM}$, food available ad libitum, room temperature of $21^{\circ} \mathrm{C} \pm 1^{\circ} \mathrm{C}$, and room humidity of $55 \% \pm$ $5 \%$. Both sexes and rats generated from at least 2 separate litters were used in all experiments. Sex was recorded at the time of tissue collection.

\section{Erythropoietin Administration}

For the chronic injury studies, 49 CCI rats, at 24 hours after injury, were randomized to receive either EPO (3000 $\mathrm{U} / \mathrm{kg}, \mathrm{R} \& \mathrm{D}$ Systems; 24 rats) or vehicle (sterile saline, 25 rats) intraperitoneally once daily on Days $1-4,6$, and 8 following CCI. The dosage of $3000 \mathrm{U} / \mathrm{kg}$ was chosen based on prior studies in preclinical models of perinatal brain injury, ${ }^{42,53,94}$ preclinical models of adult TBI, ${ }^{102}$ and human trials of both perinatal brain injury and adult TBI. ${ }^{40,77,101}$ No sham-EPO group was included because EPO has been shown to be safe in neonates after perinatal brain injury ${ }^{24,25,40,63,101}$ and in adult TBI patients. ${ }^{77}$

\section{Gait Analysis}

Computerized gait analysis (Digigait) was performed on P25-P28 rats (PID13-PID16; sham = 13 rats, CCI-veh $=11$ rats, $\mathrm{CCI}-\mathrm{EPO}=9$ rats) ${ }^{35}$ Briefly, digital video of each rat running on a backlit transparent treadmill set at $30 \mathrm{~cm} /$ sec was acquired and analyzed using Digigait software. Given the unilateral location of the CCI, ipsilateral and contralateral gait parameters were separately analyzed. Stride is a combination of the swing and stance phases. The swing phase is when the paw is not in contact with the surface. Stance refers to the period of the stride when the paw is in contact with the surface and is composed 
of a deceleration component (braking) and an acceleration component (propulsion). Braking begins at initial paw contact following the swing phase and extends to maximal paw contact, while propulsion is the period from maximal paw contact to just before the swing phase. We focused on parameters that are also evaluated in humans. Analyzed parameters included the proportion of time spent in each phase (swing, brake, propel, stance, and stride), stride length and frequency, paw area and paw force at maximal contact, stance width, gait symmetry, and ataxia.

\section{Serum and Tissue Collection}

Approximately half of each acute or chronic cohort was designated for ex vivo MRI and other histological analyses, while the other half was used for biochemical analyses. For serum assays, blood was collected on P15 or P28 and processed in a centrifuge at $6000 \mathrm{RCF}$, and serum was collected, then divided into aliquots, and stored at $-80^{\circ} \mathrm{C}$. Microdissected ipsilateral cortex samples were collected and stored at $-80^{\circ} \mathrm{C}$.

\section{Western Blot}

Samples were homogenized and sonicated in lysis buffer and processed in a centrifuge at $4200 \mathrm{RCF}$ for $10 \mathrm{~min}$. Protein concentration was determined with a Bradford assay (BioRad). Equivalent amounts of protein were loaded on $4 \%-20 \%$ Tris $\mathrm{HCl}$ gels, separated by electrophoresis, transferred to polyvinylidene fluoride (PVDF) membranes, incubated sequentially with block, primary antibody, appropriate horseradish peroxidase-conjugated secondary antibodies, and detected with chemiluminescence (Thermo). Primary antibodies were EPOR (1:100, Santa Cruz, 8 rats/group), GFAP (1:5000, AbCam, 6-12 rats/ group), and potassium chloride cotransporter 2 (KCC2; 1:100, Santa Cruz, 6-12 rats/group). Bands were quantified using an LAS 4000 imager (GE). Actin was used as a loading control for the brain, and Coomassie staining was used for serum. At least 2 blots were used to assay each protein.

\section{Ex Vivo MRI}

On P28-P35, rats (sham $=6$ rats, CCI-veh $=8$ rats, CCI-EPO $=6$ rats) were deeply anesthetized with a mixture of xylazine and ketamine and perfused with phosphate-buffered saline, followed by $4 \%$ paraformaldehyde. Brains were postfixed in $4 \%$ paraformaldehyde for 1 week and embedded in $2 \%$ agarose containing $3 \mathrm{mM}$ sodium azide for ex vivo MRI. Fixation adequately preserves brain microstructures and allows the generation of MR images with superior contrast and resolution, which is especially beneficial for evaluating white matter fibers and for assessing the brain's microstructural integrity. Magnetic resonance imaging was performed on a Bruker 4.7-T BioSpec 47/40 Ultra-Shielded Refrigerated nuclear MRI system equipped with a 72-mm inner diameter quadrature radiofrequency coil and a small-bore $(12-\mathrm{cm}$ inner diameter) gradient set with a maximum gradient strength of $50 \mathrm{Gauss} / \mathrm{cm}$. Magnetic resonance protocols consisted of multislice multiecho (MSME) spin echo and rapid acquisition with relaxation enhancement (RARE) sequences for T2-weighted imaging, susceptibility-weighted imaging (SWI), and echo-planar (EP)-DTI. Images of 12 contiguous coronal 1-mm slices were obtained (FOV $3.00 \mathrm{~cm}$, TR $2514.7 \mathrm{msec}$, TE $12 \mathrm{msec}$ ). The EP-DTI sequences used a TR of $3000 \mathrm{msec}$, TE of $40 \mathrm{msec}$, and b-value of 2000 $\mathrm{mm}^{2} / \mathrm{sec}$ with 30 gradient directions. Observers blinded to treatment status analyzed brain regions of interest (ROIs) by using Bruker's Paravision 5.1 imaging software. For this study the ROI included the ipsilateral cortex and adjacent subcortical white matter, as well as the matching contralateral regions. Tractography, fiber reconstruction, diffusion-weighted images, and FA maps were generated. The 3 eigenvectors $\left(\lambda_{1}, \lambda_{2}\right.$, and $\left.\lambda_{3}\right)$ of the diffusion tensor were measured, and ellipsoids were rendered for representative parameters using MATLAB (version 2015a, MathWorks). Fractional anisotropy, $\mathrm{MD}\left(\left[\lambda_{1}+\lambda_{2}+\lambda_{3}\right] / 3\right)$, $\operatorname{AD}\left(\lambda_{1}\right)$, and $\operatorname{RD}\left(\left[\lambda_{2}+\lambda_{3}\right] / 2\right)$ were calculated. Mean diffusivity describes the average of the 3 eigenvectors depicting the ellipsoid-shaped extent of water diffusion, whereas $\mathrm{AD}$ and $\mathrm{RD}$ provide specific detail regarding directionality. The longest primary eigenvector denotes $\mathrm{AD}$, whereas $\mathrm{RD}$ is the mean of the 2 smaller eigenvectors. Given our resource limitations and our observation from functional studies that sex had an insignificant impact on outcome, separate analyses by sex were not performed for the DTI studies.

\section{Multiplex Determination of Cytokine Expression}

Serum concentrations of inflammatory cytokines were determined in duplicate ${ }^{98}$ with the aid of a V-PLEX multielectrochemiluminescence (MECI) immunoassay (MesoScale Discovery) for 9 cytokines: CXCL1 (known as CINC in the rat), interferon gamma (IFN $\gamma$ ), interleukin1beta (IL-1 $\beta$ ), IL-4, IL-5, IL-6, IL-10, IL-13, and TNF $\alpha$ at the acute stage (P15, 6 rats/group) or at P25-P40 (6-11 rats/group). Serum (1:4 dilution) or calibrator was loaded onto a multispot plate in duplicates, and assays were performed per the manufacturer's protocol. Plates were read on a Quickplex SQ 120 Imager. The coefficient of variation was less than $15 \%$ for each analyte, and interassay variation was less than $15 \%$ when samples were retested across plates to check assay reliability.

\section{Statistical Analysis}

The Shapiro-Wilk test was used to test each group of data for normality, and Levene's test was used to check the homogeneity of variances. For analysis of 2 groups at 1 age (sham vs CCI), a 2-tailed Student t-test with unequal variance or the Mann-Whitney U-test was performed, as appropriate. For analysis of more than 2 groups (sham, CCI-veh, and CCI-EPO), a 2-way ANOVA (injury $\times$ treatment) was performed with Bonferroni's post hoc correction for multiple comparisons using SPSS version 21 (IBM Corp.). For all analyses, $\mathrm{p}<0.05$ was considered significant.

\section{Results}

\section{Clinical Manifestations of Infant CCI}

In this study mortality was $4.8 \%(6 / 125,5 \mathrm{CCI}$ and 1 sham) within 1 hour of the procedure. No mortality oc- 
curred after 1 hour, and no animals suffered infection. After injury, most rats appeared somnolent, in contrast to the shams, which were rapidly alert. Moreover, 42 (55\%) of 76 injured pups were noted to display intermittent head nodding and shaking suggestive of postimpact seizure activity that began within an hour of their injury and persisted for up to 2 days before resolving. Four $(8.1 \%)$ of 49 injured rats that were designated for chronic analyses (1 CCI-veh and 3 CCI-EPO) developed cranial protuberances suggestive of a leptomeningeal cyst $6-12$ days following the CCI. To confirm that the protuberances were attributable to $a b-$ normal CSF dynamics and not infection, we used MRI with T2-weighted and SWI sequences. Deformation of the lateral ventricle toward the pia, consistent with a leptomeningeal cyst, was observed on the MR images (Fig. 2A). Despite our best efforts to avoid dural injury during the craniectomy and impact, a dural laceration probably occurred in this small proportion of injured animals. These 4 brains were not included in the subsequent analyses. Altogether, this P12 impact model appears to replicate multiple clinical components of infantile TBI, including an alteration in consciousness, early postimpact seizures, and, in a small subset, the formation of a leptomeningeal cyst.

\section{Structural Injury Varying From Moderate to Severe on SWI}

To determine the variation in the severity of the structural injury and the degree of hemorrhage, SWI was per-
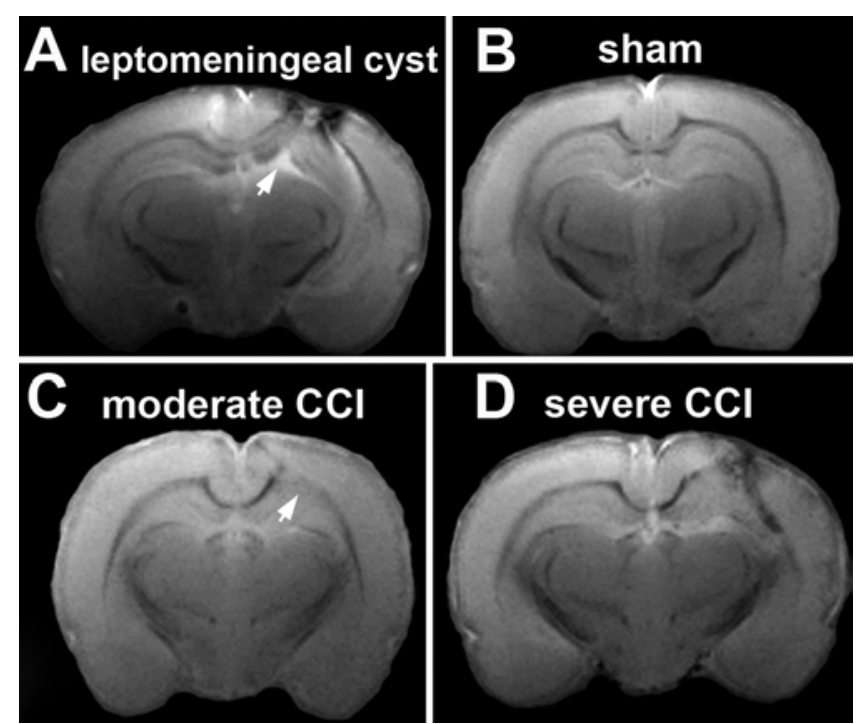

FIG. 2. Representative SWI studies of sham and $\mathrm{CCI}$ brains reveal the spectrum of sustained injury from $\mathrm{P} 12$. A: A few $\mathrm{CCl}$ rats developed a cranial protuberance suggestive of a leptomeningeal cyst $6-12$ days after $\mathrm{CCl}$. Susceptibility-weighted imaging demonstrated deformation of the lateral ventricle toward the cortical lesion (arrow), similar to the pattern observed in human infants with a leptomeningeal cyst. B: Sham rats demonstrated no abnormalities in the cortex and sharp demarcation of the underlying subcortical white matter. C: In contrast, $\mathrm{CCl}$ rats demonstrated a spectrum of injury, with approximately half of the $\mathrm{CCl}$ rats showing moderate injury with the loss of the gray-white matter demarcation (arrow) and mild extension of hypointense signal into the overlying cortex. D: The remaining half of the $\mathrm{CCl}$ rats that underwent SWI had severe injury with extensive focal injury to the cortex, subcortical white matter, and dorsal hippocampus. formed 2-3 weeks following impact at P28-P35. None of the 6 sham brains had any evidence of injury or hemorrhage (Fig. 2B). Of the $14 \mathrm{CCI}$ brains subjected to SWI, the extent of injury varied along a spectrum from moderate to severe. Seven CCI brains showed moderate injury primarily limited to the white matter with minimal to moderate extension to the overlying cortex (Fig. 2C). Injury consisted of the loss of sharp white matter definition, and hypointense signal suggested hemorrhage. The other half of the CCI brains had severe injury that involved the overlying cortex and white matter and often extended to the dorsal hippocampus with obvious structural deformation (Fig. 2D). These results show that SWI offers an approximation of the extent of injury and hemorrhage, with findings similar to those found in humans.

\section{Infant $\mathrm{CCI}$ Increasing EPOR Expression at PID3}

To identify potential responsiveness to EPO treatment in the infant brain following impact injury, EPOR levels were quantified using immunoblotting in the ipsilateral cortex at P15, that is, 3 days postinjury when the damage was probably near maximal. At PID3, EPOR expression was increased by $30 \%$ in the CCI-veh cortex (8 rats) compared with shams ( 8 rats, $p=0.008$; Fig. 3 ). These data show that the developing brain responds to impact TBI with an increase in EPOR expression, suggesting that the injured developing CNS should be responsive to exogenous EPO administration.

\section{Erythropoietin Treatment and Subtle Motor Deficits Following Infant $\mathrm{CCl}$}

To determine whether EPO treatment alters chronic motor function, detailed digital gait analyses were performed on P25-P28 juvenile rats, approximately 2 weeks after a CCI (PID13-PID16). The CCI-veh rats (11 ani-
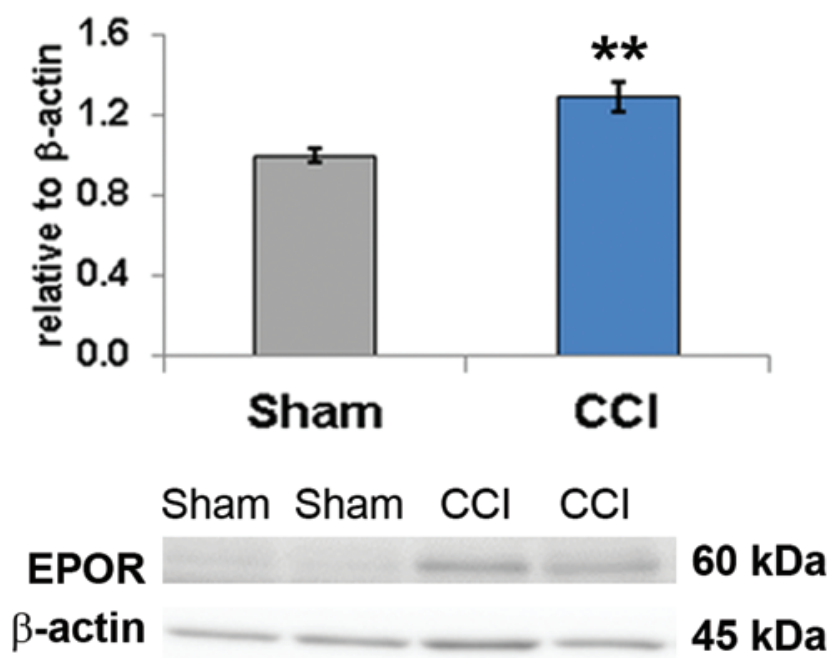

FIG. 3. Immunoblotting of EPOR protein in microdissected cortex ipsilateral to the impact revealed that $\mathrm{CCl}$ induces a significant increase in EPOR expression 3 days after injury and suggests that vacant CNS EPOR should be responsive to exogenous EPO treatment days after injury. $\mathrm{CCl}=\mathrm{CCl}$-veh. ${ }^{* *} \mathrm{p}=0.008$, 2-tailed t-test with unequal variance. Figure is available in color online only. 
mals) showed a mildly altered gait pattern in the forelimb ipsilateral to the injury compared with shams (13 animals) or CCI-EPO rats (9 animals). Specifically, the left forelimb in CCI-veh rats spent proportionately less time in the brake phase of the stance than that in the shams $(p=0.02)$ or CCI-EPO rats ( $p=0.003$; Fig. $4 \mathrm{~A})$. In a compensatory manner, the left forelimb in CCI-veh rats spent a greater proportion of time in the propel phase of the stance than that in shams $(p=0.02)$ or CCI-EPO rats $(p=0.003$; Fig. 4B). In addition, the forelimb stance width was narrower in the CCI-veh rats than in the shams $(p=0.004)$ or CCI-EPO rats ( $p=0.001$; Fig. $4 \mathrm{C}$ ). In this study no other significant differences in gait were present in the other 3 limbs among sham, CCI-veh, and CCI-EPO rats (data not shown). Despite the relatively severe injury to the left hemisphere with neurological signs at the time of injury, the motor deficit in the subacute period 2 weeks after CCI injury was relatively mild.

\section{Erythropoietin Treatment and DTI Abnormalities From CCI}

Diffusion tensor imaging was performed to more specifically quantify the extent of injury and the potential efficacy of EPO treatment. To determine if DTI, which is used to measure microstructural injury in humans, could also quantify chronic injury in infant rats following CCI, rat brains were imaged (PID16-23) at P28-P35, an age equivalent to a juvenile human. Color maps showed a loss of directionality in the region of direct impact in the ipsilateral cortex and white matter and in the contralateral white matter, with improvement following EPO treatment (Fig. 5A). Ellipsoids were plotted using the mean of each eigenvector $\left(\lambda_{1}, \lambda_{2}\right.$, and $\left.\lambda_{3}\right)$ for a region of interest to determine whether all 3 eigenvectors were independently changed by injury and EPO treatment. Representative ellipsoids show that diffusivity increases in all directions in the ipsilateral cortex in the CCI-veh brains compared with shams (Fig. 5B). By contrast, diffusivity in the CCI-EPO cortex is similar to that in shams and is reduced in all directions compared with CCI-veh cortex. Detailed analyses of DTI parameters revealed widespread microstructural abnormalities involving the ipsilateral lesion cortex and white matter, as well as the contralateral cortex and white matter. Specifically, in CCI-veh brains (8 rats), the ipsilateral lesion cortex FA was reduced compared with that in sham brains ( 6 rats, $\mathrm{p}=$ 0.004, 2-way ANOVA; Fig. 6A), showing a loss of microstructural integrity. Similarly, the ipsilateral white matter FA was reduced ( $p<0.001$; Fig. 6B). The FA was also lower in the contralateral hemisphere white matter in CCI-veh brains ( $p=0.001$; Fig. $6 C$ ), suggestive of widespread white matter injury that extended into the contralateral hemisphere. By contrast, only a minor reduction in FA occurred in the contralateral cortex $(\mathrm{p}=\mathrm{NS}$; Fig. 6D). Collectively, these findings showed that P12 CCI caused widespread loss of structural integrity affecting both hemispheres.

A detailed analysis of diffusivity parameters, measures that have also been used to assess TBI in humans, was performed to determine whether EPO treatment altered microstructural recovery from infant CCI. In the chronic phase, CCI-veh lesion cortex MD was elevated compared with that in sham $(\mathrm{p}=0.011)$ and CCI-EPO brains $(\mathrm{p}=$ 0.04; Fig. 7A), and MD levels in the sham and CCI-EPO brains were similar to one another, suggesting that MD was responsive to EPO treatment. To more specifically define the direction of the altered diffusivity present, AD, the primary eigenvector, and RD, the mean of the 2 smaller eigenvectors, were measured. In CCI-veh lesion cortex, the AD was increased compared with that in shams ( $p$ $=0.014)$ and was reduced to sham levels following EPO treatment ( $p=0.020$; Fig. 7B). Similarly, RD was also elevated in the CCI-veh lesion cortex compared with that in shams $(p=0.01)$ and improved to sham levels with EPO treatment $(p=0.037$; Fig. $7 C$ ). A similar pattern for $\mathrm{MD}$, $\mathrm{AD}$, and $\mathrm{RD}$ was observed in the ipsilateral white matter (Fig. 7D-F). In summary, these results showed that an extended course of EPO treatment following P12 CCI normalizes the chronic changes in $\mathrm{MD}, \mathrm{AD}$, and $\mathrm{RD}$ in the lesion cortex and adjacent white matter. Together, these data showed that the chronic ipsilateral microstructural injury that altered water diffusion improved with EPO treatment.

To determine if EPO treatment also affected widespread injury in the contralateral hemisphere, diffusivity parameters were evaluated in the contralateral white matter and cortex corresponding to the lesion site. In the contralateral white matter, MD was also increased in CCI-veh brains compared with that in shams $(\mathrm{p}<0.001$; Fig. $7 \mathrm{G})$ almost to the same extent as in the ipsilateral white matter, sug-
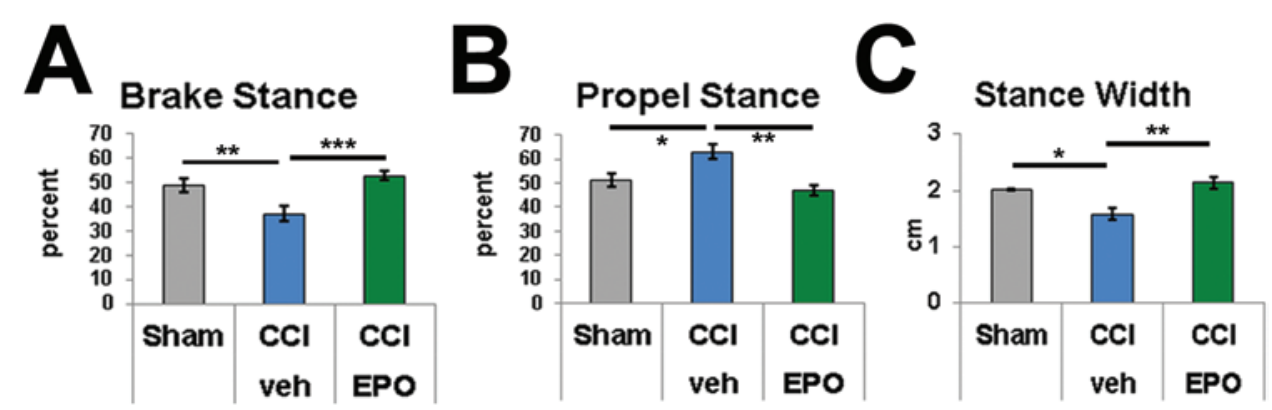

FIG. 4. Controlled cortical impact on $P 12$ disturbed gait patterns, and extended EPO treatment reversed gait abnormalities. A: The portion of the stance spent in the brake phase was significantly reduced in $\mathrm{CCl}$-veh rats, and EPO treatment restored the brake portion to sham levels. B: Similarly, the portion spent in the propel phase was increased following CCI and normalized with EPO treatment. C: The forelimb stance width was also significantly reduced in $\mathrm{CCl}$ rats compared with shams and normalized with EPO treatment. ${ }^{*} p<0.05,{ }^{* *} p<0.01,{ }^{* * *} p \leq 0.001,2$-way ANOVA with Bonferroni post hoc correction. Figure is available in color online only. 


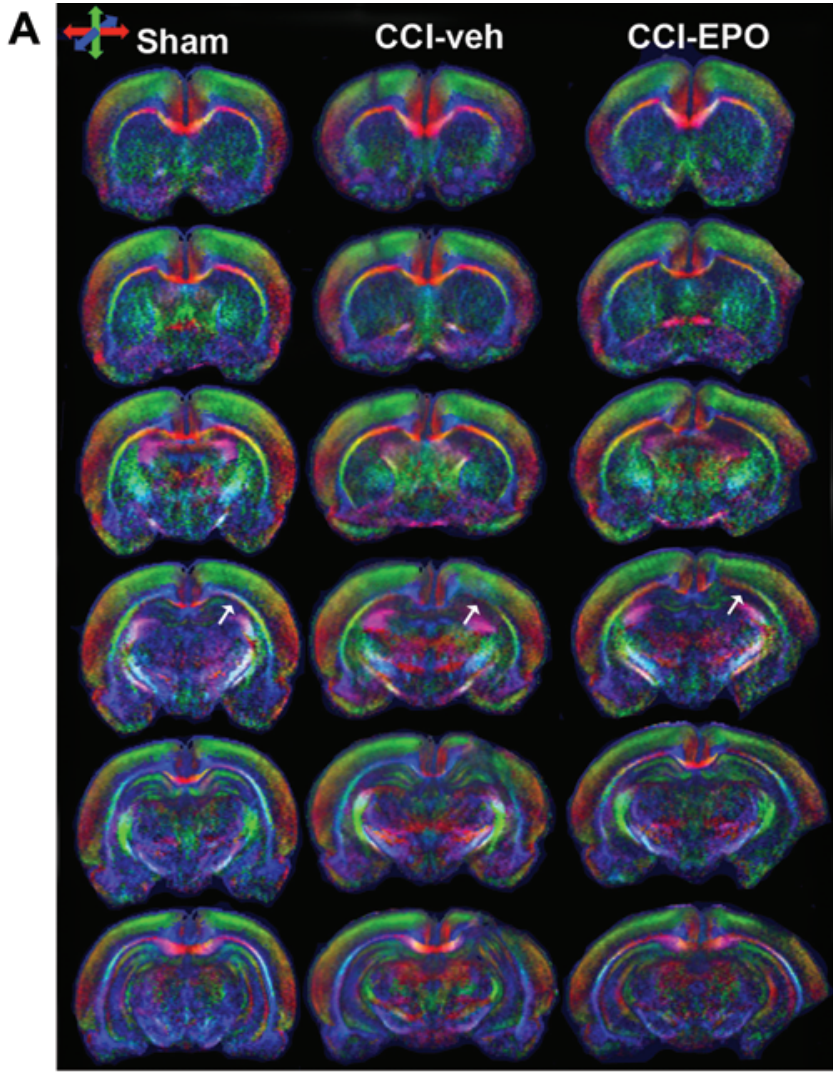

B

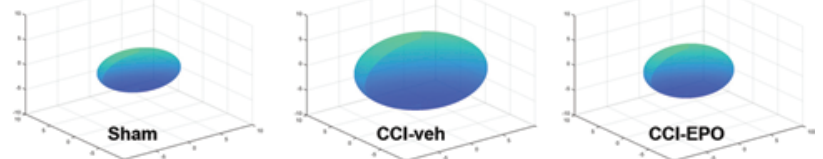

FIG. 5. Diffusion tensor imaging measures the diffusion of water in the CNS. Diffusion is constrained by structures such as myelin, axons, and cellular organelles, and injury alters the diffusivity of water. A: Color maps of representative sham, $\mathrm{CCl}$-veh, and $\mathrm{CCl}$-EPO rat brains. Note that the color intensity is reduced in the region of the $\mathrm{CCl}$ (arrows), indicative of a loss of microstructural integrity. Improvement is apparent in the $\mathrm{CCI}$-EPO brain. Directionality of DTI is indicated by different colors: red, transverse; green, vertical; and blue, orthogonal plane. B: The average of each of the 3 eigenvectors $\left(\lambda_{1}, \lambda_{2}\right.$, and $\left.\lambda_{3}\right)$ for a ROI can be modeled by an ellipsoid to show directional changes in diffusivity after injury or repair. Ellipsoids representing the mean eigenvectors for the ipsilateral cortex for sham, $\mathrm{CCl}$-veh, and $\mathrm{CCl}-\mathrm{EPO}$ rats demonstrate that diffusivity increases after injury in all directions in $\mathrm{CCl}$-veh rat brains and that EPO treatment reduces diffusivity to levels similar to those in shams. Figure is available in color online only.

gesting extensive white matter injury. Notably, the white matter damage represented by MD improved with EPO treatment $(p=0.005)$, suggesting that EPO treatment affects widespread, extensive white matter injury. As in the ipsilateral white matter, AD increased in the contralateral white matter compared with that in shams $(p=0.001)$, and the injury was reduced with EPO treatment $(p=0.002$; Fig. $7 \mathrm{H}$ ). Radial diffusivity in the contralateral white matter was also elevated in CCI-veh brains compared with that in shams $(\mathrm{p}<0.001)$ and improved with EPO treatment $(\mathrm{p}$ $=0.01$; Fig. 7I). Like the widespread injury evident in the contralateral white matter, a similar pattern was observed in the contralateral lesion cortex. The $\mathrm{MD}, \mathrm{AD}$, and $\mathrm{RD}$ of the contralateral lesion cortex were elevated in the CCIveh brains and improved with EPO treatment (Fig. 7J-L). In summary, P12 CCI induced widespread microstructural injury to the contralateral white matter and overlying cortex, as shown by changes in MD, AD, and RD. Moreover, an extended course of EPO treatment reversed this damage, suggesting that the impact of EPO therapy may repair the widespread, extensive chronic injury to white matter that occurs in the developing brain after impact injury. Together, these results suggest that diffusivity may provide an MRI biomarker to detect sustained improvement during recovery after impact TBI.

\section{Infant $\mathrm{CCl}$ Coinciding With Excess Calpain Degradation in Brain}

In addition to identifying imaging biomarkers, we also sought to identify serum biomarkers of TBI, as many infants with severe TBI are too ill to be frequently transported to an MRI suite. While serum biomarkers lack the localization provided by MRI, serum levels of these markers may reflect ongoing cellular injury or recovery and are amenable to more frequent analysis without the need for patient transport. Candidate serum biomarkers for TBI include inflammatory cytokines and calpain DPs of CNS molecules.

Since excess calpain activity has not been documented in this model of infant TBI, we first investigated whether excess DPs were present acutely in the lesioned cortex, that is, PID3. Activated calpain degrades full-length $\alpha$ II-spectrin (approximately $250 \mathrm{kD}$ ), a neuron-specific cytoskeletal protein, into a calpain-specific $145-\mathrm{kD}$ fragment, and the ratio of cleaved to full-length $\alpha$ II-spectrin DPs ( $\alpha$ II-SDPs) is a measure of calpain activity. In P15 rats, calpain-induced $\alpha$ II-SDPs were elevated 2.2-fold in the cortex following CCI (PID3, 12 rats) compared with levels in shams (10 rats, $p<0.001$, 2-tailed t-test; Fig. 8A). Because neuronspecific KCC2 promotes multiple components of neuronal function and is also a calpain target, brain KCC2-DPs may also reflect neuronal health after injury. Three days after CCI, 90-kD KCC2-DP levels in CCI-veh cortex (12 rats) were elevated compared with sham levels (10 rats, $p=$ 0.037; Fig. 8B). The astrocyte filament GFAP is a marker of gliosis and a substrate of calpain. The sum of full-length GFAP and calpain DPs reflects the amount of gliosis, while DP levels alone probably represent the degree of injury mediated by excess calpain activity. We hypothesized that 3 days after CCI in P12 rats both the sum total expression of GFAP due to gliosis and the amount of GFAP-DPs due to excess calpain activity would increase. As expected, the sum total GFAP levels increased 2-fold in the CCI-veh cortex (6 rats) compared with sham levels $(6, p=0.005$; Fig. 8C). Levels of GFAP-DPs also increased at PID3 compared with sham levels $(p=0.002)$. Together, these results show that, 3 days after injury, infant CCI precipitates excess calpain activity at least in the region of injury and alters the levels of $\alpha$ II-SDPs, KCC2-DPs, and GFAP-DPs.

\section{Serum Biomarker Levels Altered by Infant CCI}

Since excess serum calpain activity is a biomarker that 

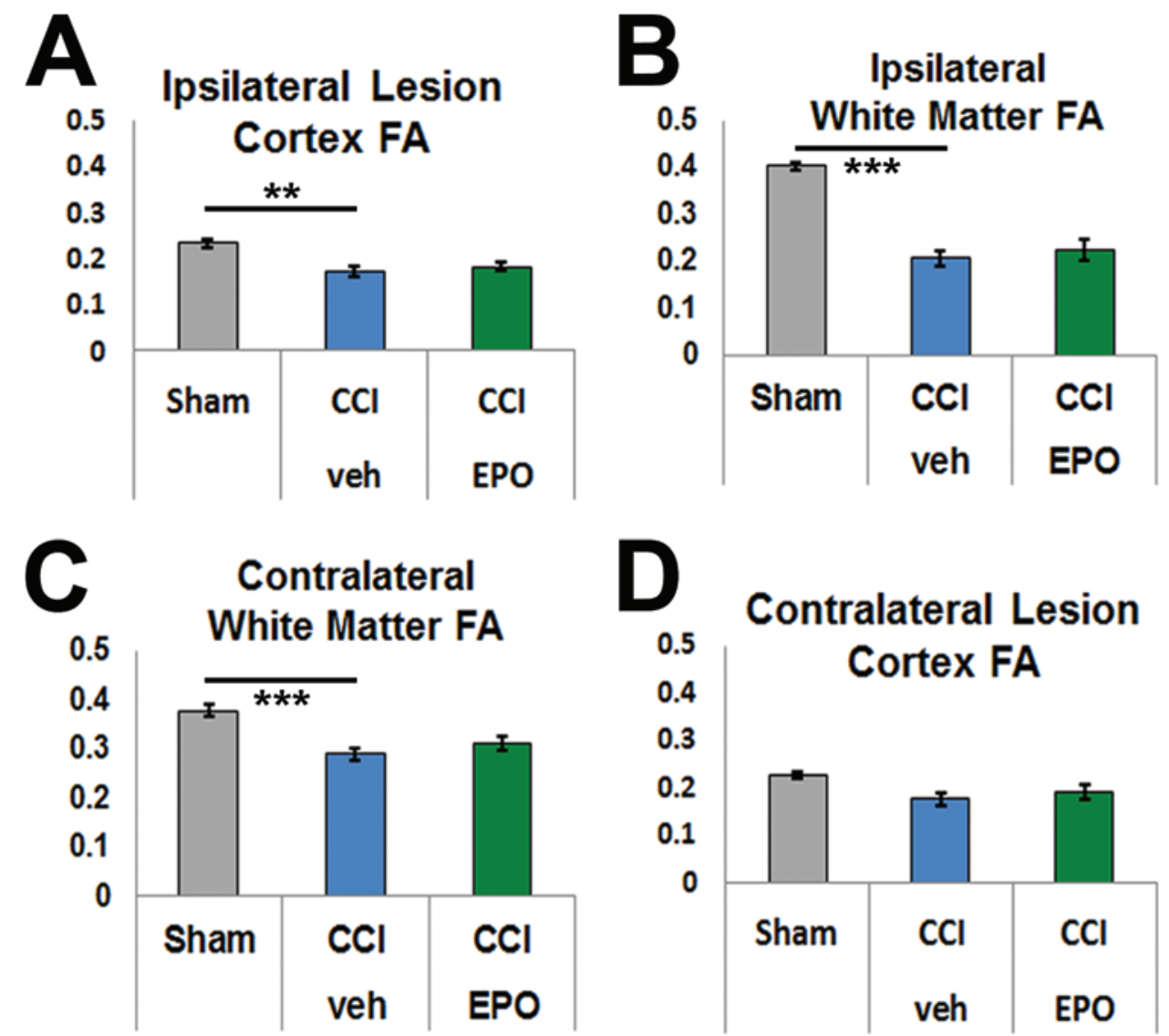

FIG. 6. Ipsilateral cortex and bilateral subcortical white matter show loss of FA, a crude measure of microstructural integrity. A: In the ipsilateral cortex, FA was significantly reduced in $\mathrm{CCI}$ brains. B: Similarly, FA was significantly lower in the ipsilateral subcortical white matter. $\mathrm{C}$ : The reduction in FA following $\mathrm{CCl}$ extended to the contralateral subcortical white matter. D: By contrast, the FA in the contralateral cortex was not affected. ${ }^{*} p<0.05,{ }^{* *} p<0.01,{ }^{* * *} p \leq 0.001$, 2-way ANOVA with Bonferroni post hoc correction. Figure is available in color online only.

could potentially be measured in human infants following TBI, we determined whether the observed excess calpain activity in the brain following infant CCI was also detectable in the serum. Serum levels of total GFAP and GFAPDPs were quantified at P15 using immunoblotting. The sum total of GFAP was elevated in the serum from CCIveh rats (7 rats) compared with sham levels (8 rats, $\mathrm{p}<$ 0.001; Fig. 9A). Similarly, GFAP-DPs were elevated in the serum from CCI-veh rats compared with sham levels ( $\mathrm{p}=$ 0.001; Fig. 9B). These data show that serum GFAP levels reflect the same pattern of calpain degradation observed in the CNS following infant CCI.

Serum cytokine levels have also been suggested as potential serum biomarkers. Nine serum inflammatory cytokines were surveyed at P15 using the MECI immunoassay. At P15 serum TNFo levels from CCI-veh rats (6 rats) were elevated compared with sham levels ( 6 rats, $p=0.01$; Fig. 9C). In chronic samples from P25-P40, no difference in serum TNF $\alpha$ levels was present when shams (10 rats) were compared with either CCI-veh (11 rats) or CCI-EPO (6 rats; Fig. 9D) rats, suggesting that TNF $\alpha$ was elevated in the serum only in the acute period following CCI. Interestingly, serum CXCL1 levels (6 rats) were elevated at P15, compared with sham levels (6 rats, $p=0.009$; Fig. 9E). Moreover, serum CXCL1 levels were persistently elevated weeks after CCI at P25-P40 (11 rats) compared with levels in shams (10 rats, $p=0.033$, 2-way ANOVA; Fig. 9F). Most importantly, serum CXCL1 levels were modulated by an extended course of postinjury EPO treatment (6 rats, $\mathrm{p}=$ 0.022; Fig. 9F). In contrast, at either P15 or P25-P40, serum levels for the remaining cytokines were either below the level of detection (IL-1 $\beta$ and IL-5) or no differences between shams and CCI-veh rats were detected (IFN $\gamma$, IL4, IL-6, IL-10, and IL-13). Thus, the elevations observed in serum TNF $\alpha$ and CXCL1 levels suggest that specific signaling mechanisms are activated rather than a general inflammatory response.

\section{Discussion}

Our overall goal is to translate diagnostic and therapeutic strategies from a preclinical model to infants who suffer moderate to severe TBI. Our first objective with this initial study was to determine whether emerging human clinical trial-compatible MRI and serum biomarkers could detect injury in a clinically relevant rodent model of infant impact TBI. The second objective was to test whether the neurorestorative cytokine EPO, administered in an extended dosing paradigm congruent with its mechanisms of action, could demonstrate efficacy in infant TBI. We found that 

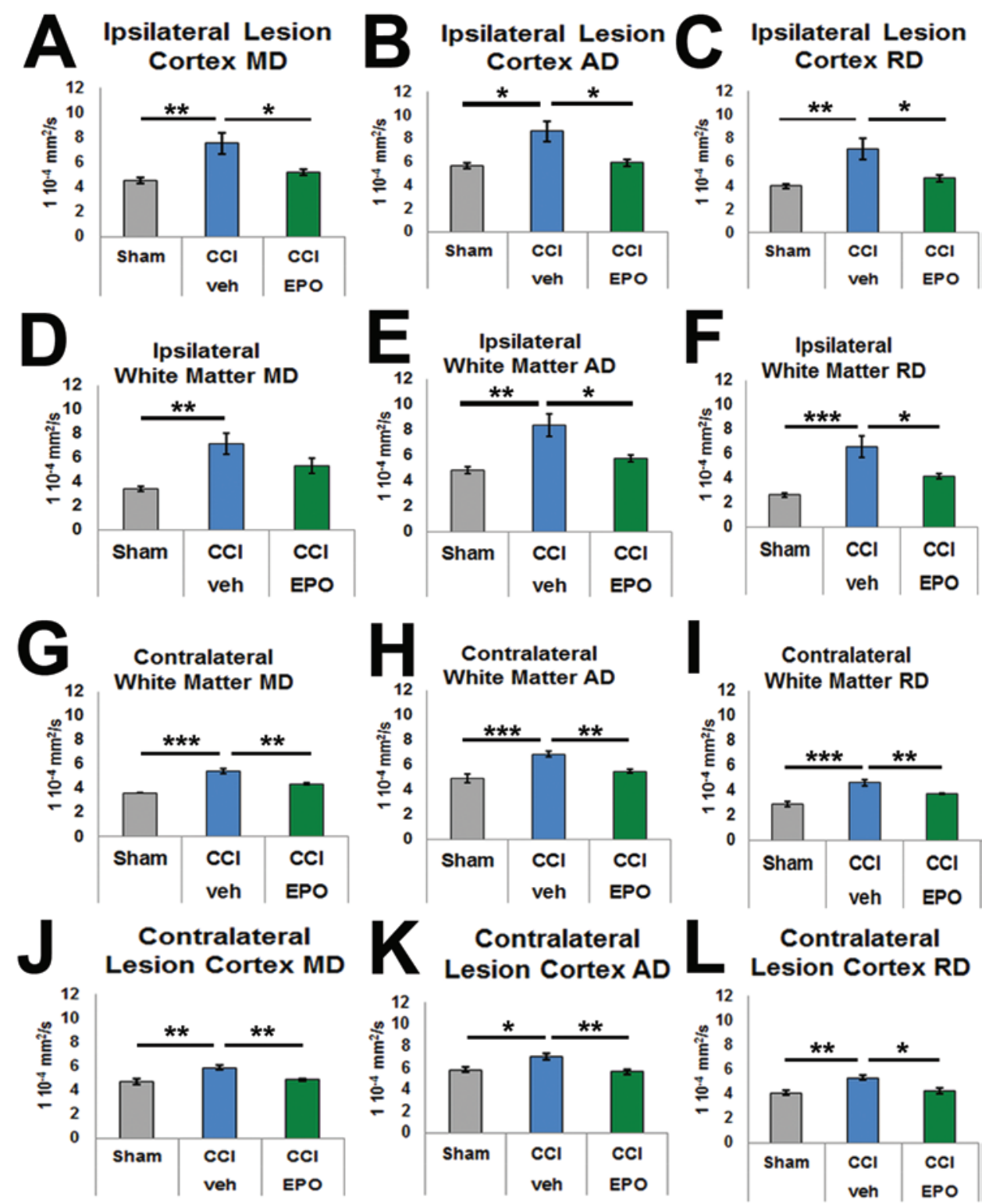

FIG. 7. Detailed analyses of DTI eigenvectors demonstrated significant efficacy of extended EPO treatment following P12 CCI, providing MRI biomarkers of treatment responsiveness. Mean diffusivity describes the average of the 3 eigenvectors depicting the ellipsoid-shaped extent of water diffusion, whereas AD and RD provide specific detail regarding directionality. The longest, primary eigenvector denotes $\mathrm{AD}$, whereas $\mathrm{RD}$ is the mean of the 2 smaller eigenvectors. In CCl-veh brains, MD was elevated in the ipsilateral lesion cortex $(\mathbf{A})$ and subcortical white matter $(\mathbf{D})$. More specifically, $A D$ was elevated in both the lesion cortex $(\mathbf{B})$ and the subcortical white matter (E). Similarly, RD was also increased in both areas (C and F). Most importantly, CCI-EPO brains showed significant restoration of $A D$ and $R D$ in both brain regions. As expected, the magnitude of the increase in diffusivity after injury was less prominent in the contralateral subcortical white matter and cortex $(\mathbf{G}-\mathbf{L})$. The efficacy of EPO treatment was similar to that in the ipsilateral hemisphere, indicating that after injury extended EPO treatment imparts widespread microstructural restoration. ${ }^{*} p<$ $0.05,{ }^{* *} p<0.01,{ }^{* * *} p \leq 0.001,2$-way ANOVA with Bonferroni post hoc correction. Figure is available in color online only.

P12 rats, approximately equivalent to 6-month-old human infants, subjected to CCI developed an acute clinical presentation similar to that of human infants with severe impact TBI. Controlled cortical impact on P12 produces an acute elevation in cortical EPOR expression, accompanied by excess calpain activity and a specific serum inflammatory cytokine response. The CCI also causes a chronic functional motor deficit in juvenile rats that is accompanied by chronic SWI and DTI abnormalities, as well as a chronic elevation in serum CXCL1 levels. Importantly, these im- aging and serum biomarkers demonstrated responsiveness to EPO treatment-the first time that treatment responsiveness using human-compatible biomarkers has been shown in a model of infant TBI.

\section{Clinical Manifestations of Infant TBI and EPO Treatment}

The pattern of response to the P12 CCI injury with a somnolent appearance and shaking suggestive of postimpact seizures is congruent with the clinical pattern often described for infants who reportedly suffer an impact- 


\section{A \\ P15 Cortex \\ $\alpha$ ll-spectrin Ratio}

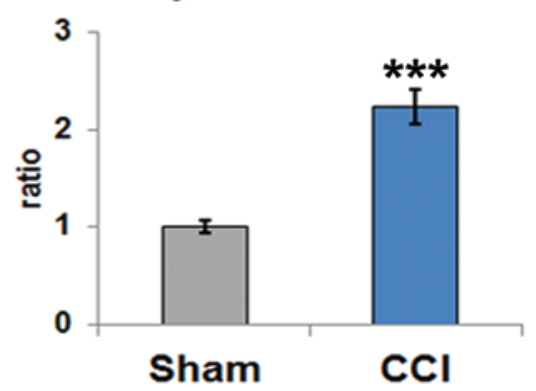

Sham CCI

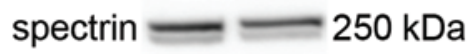

$145 \mathrm{kDa}$

$\beta$-actin

\section{P15 Cortex GFAP Sum}

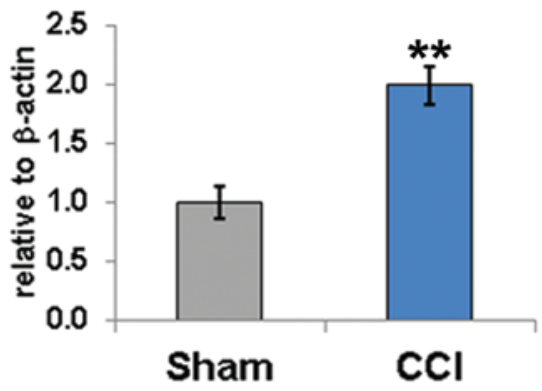

B

P15 Cortex

KCC2 90 kDa

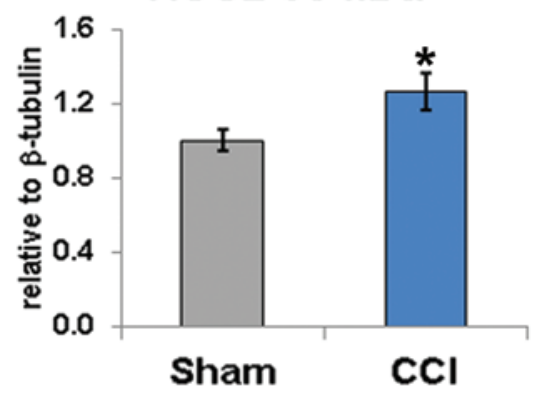

Sham $\mathrm{CCl}$

KCC2 $\quad 90 \mathrm{kDa}$

$\beta$-tubulin
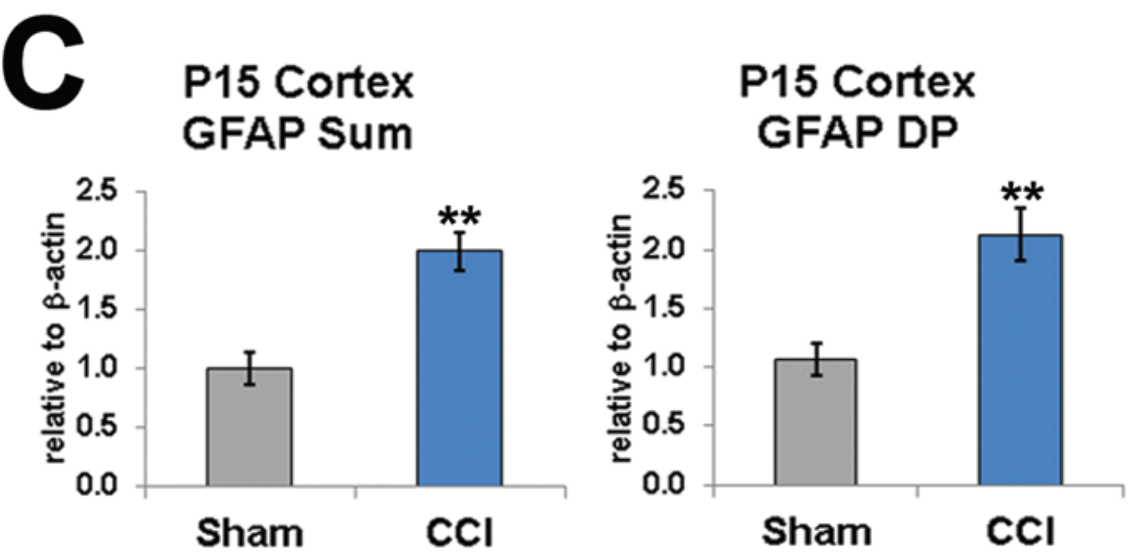

P15 Cortex GFAP DP

Sham $\mathrm{CCl}$

GFAP

55 kDa

\section{$\beta$-actin}

FIG. 8. Excess cortical calpain activity at P15 degrades essential neuromolecules. A: The ratio of $\alpha$ ll-SDPs, a neuron-specific cytoskeletal protein, was significantly elevated at PID3. B: Calpain DPs for KCC2, a molecule important for neuronal function, were also significantly increased at PID3. C: The astrocyte intermediate GFAP increases proportionally with gliosis and is also degraded by calpain. After CCI, the sum total of GFAP levels (left) and the GFAP-DPs (right) were both significantly increased. ${ }^{*} \mathrm{p}$ $<0.05,{ }^{* *} p<0.01,{ }^{* * *} p \leq 0.001,2$-tailed t-test with unequal variance. Figure is available in color online only.

induced head injury. This injury response is also consistent with those exhibited in other infant and toddler TBI models that include a description of the initial injury response. ${ }^{3,106}$ Chronic posttraumatic epilepsy is a significant problem for infants who suffer severe TBI, ${ }^{6,64}$ and detailed analyses of the alterations in the chronic seizure threshold using this CCI model are underway. All rats in our study received injuries of the same depth of impact yet demonstrated a spectrum of structural injury, similar to the range of injury observed in infant humans with known impact injuries. While larger mammals mimic infant humans bet- ter in the ratio of gray to white matter, an important factor when studying injury to the developing brain, ${ }^{106}$ rats are less resource intensive than larger mammals. In summary, this rat model of CCI on P12 allows testing of specific hypotheses focused on translation to clinical trials of interventions in infant humans.

Erythropoietin signaling in neural cells invokes multiple mechanisms of repair that are manifested over an extended timeframe of at least several days, including the genesis, survival, and maturation of neurons and oligodendrocytes, ${ }^{14,38,53,90,97}$ as well as reduces calpain degradation 

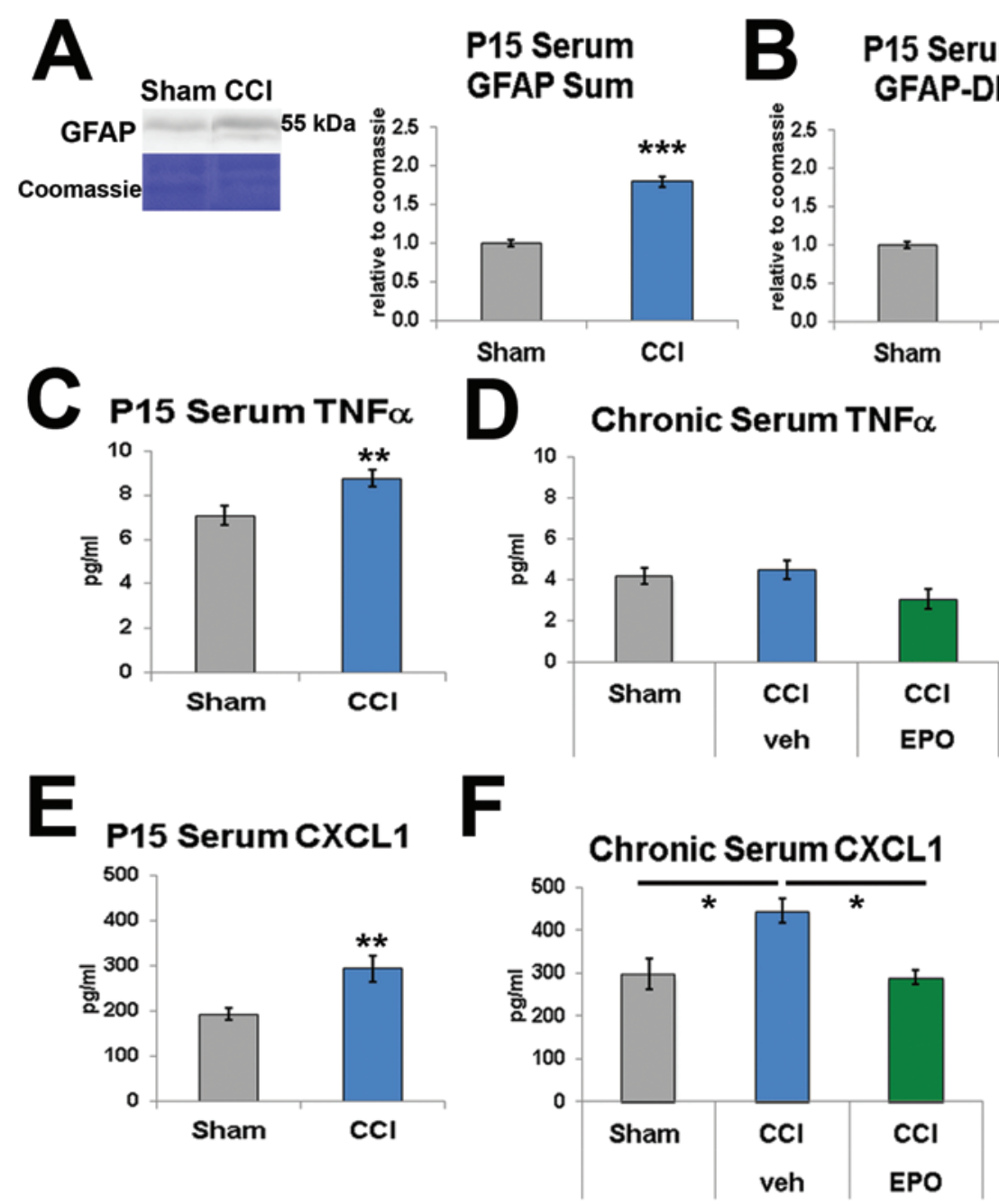

FIG. 9. Serum biomarkers reflect CNS injury patterns. A: Serum GFAP levels were assayed with immunoblotting using Coomassie staining as a loading control, and the sum total of full-length and DPs (approximately $48 \mathrm{kD}$ ) was significantly elevated in serum 3 days after injury. B: Similarly, GFAP-DP levels were significantly increased at P15. C: Serum TNF $\alpha$ levels were significantly increased at P15. D: Serum TNF $\alpha$ levels normalized by the chronic phase. E: Serum CXCL1 (known as CINC in the rat) levels were also significantly increased at PID3. F: Serum CXCL1 levels remained elevated in the chronic phase and, importantly, were normalized by an extended course of EPO treatment. $\mathrm{CCl}=\mathrm{CCl}$-veh. ${ }^{*} \mathrm{p}<0.05,{ }^{* *} \mathrm{p}<0.01,{ }^{* * *} \mathrm{p} \leq 0.001$, 2-tailed t-test with unequal variance $(A-C, E)$ and 2-way ANOVA with Bonferroni post hoc correction (D and F). Figure is available in color online only.

and chronic inflammation. ${ }^{29,37,39}$ These mechanisms of action support the rationale that an extended dosing regimen with neuroprotective doses of EPO is probably necessary to alter recovery after TBI. In particular, because exogenous EPO promotes oligodendroglial lineage survival and maturation $^{38}$ and because recovery of the oligodendroglial lineage extends over 3 months after CCI injury in mice, ${ }^{19} \mathrm{EPO}$ treatment should theoretically benefit white matter recovery over a protracted period. In extended, neuro-reparative dosing regimens, EPO has shown promise in adult trials of other disease processes ${ }^{60,61}$ Moreover, EPO treatment has shown benefit in clinical trials of preterm infants both through improved early long-term imaging ${ }^{46}$ and through improved long-term cognitive outcomes at 2 years. ${ }^{63}$ Conversely, in a recent US trial of adults with severe nonpenetrating TBI, EPO treatment did not provide significant benefit $;{ }^{77}$ however, the trial was underpowered to detect an effect using higher neuro-reparative doses. Another recent, very small trial from Turkey suggested improvement with a version of extended EPO treatment, ${ }^{4}$ though this study was similarly underpowered. In a preclinical model of toddler TBI in rats with CCI on P17, extended EPO dosing was effective in reducing neuronal apoptosis and improving novel object recognition at 14 days after injury. ${ }^{85}$ A longer duration of EPO treatment provided additional functional improvement in adult CCI rats. ${ }^{102}$ In the present study, the dosing regimen was designed to be extended and to use high neuro-reparative doses. Importantly, in clinical trials, high-dose EPO treatment has been safe for infants with perinatal brain injury $25,55,101$ and adults with TBI.77 Additional studies are needed to determine whether EPO repair is dose dependent. In the present study 6 doses were given over 8 days following CCI. Elevated EPOR levels were found in the injured cortex 3 days after CCI, but the 
regional and temporal patterns of excess EPOR expression have yet to be fully characterized. This first approximation at an extended dosing regimen for infant TBI demonstrated efficacy with improvement in motor function, as well as imaging and serum biomarkers.

Controlled cortical impact rats showed a subtle motor deficit with a shift in the proportion of time spent during stance from the brake phase to propel phase in the forelimb ipsilateral to the CCI. The CCI-veh rats showed a narrower stance width, which is associated with instability in humans. ${ }^{22}$ These relatively subtle deficits are markedly different from the severe gait deficits found in prior computerized gait analyses performed in same-age rats (P25P28) with different types of early CNS injury, 34,35 which argues that the testing paradigm is capable of detecting complex gait deficits. Motor deficits may be subtle because the primary point of impact was in the parietal region and not in the motor strip. The lack of a severe chronic motor deficit also reflects injury to the brain at this particular stage of development. Significantly, in infant humans motor deficits can be subtle on clinical examination despite the presence of significant anatomical abnormalities. For example, infants with prenatal middle cerebral artery stroke may not manifest motor deficits until a year later. ${ }^{45}$ Though a mouse model of frontal lobe CCI injury to a toddler-age equivalent (P21) has shown a chronic motor deficit, ${ }^{17}$ no other preclinical models of infant or toddler TBI have shown chronic motor deficits following early CCI. ${ }^{1,84,106}$ Thus, even though the deficits detected in the present study were subtle, this is the first time that chronic significant gait differences in infant CCI-veh rats were identified. Most importantly, EPO treatment reversed the motor deficits. Testing of other functional deficits, specifically cognitive deficits, social interaction, and impulsivity, has not yet been performed in this model but is anticipated. In a Morris water maze, infant P11 CCI rats performed worse than toddler P17 CCI rats ${ }^{74}$ which suggests that cognitive deficits are likely to be found in our P12 CCI rats. In addition, in a toddler P17 model of TBI, EPO treatment restored memory testing novel object recognition in rats at 2 weeks after injury. ${ }^{85}$ Here, EPO treatment restored motor performance, demonstrating that extended postinjury EPO treatment can induce sustained improvement following infant TBI.

\section{Imaging Biomarkers Reflect Functional Improvement With EPO Treatment}

In the present study widespread injury extending beyond the area of impact was found on T2-weighted and SWI of chronic injury, consistent with the extensive insult beyond the impact region that others have found following early CCI using traditional histological analyses. ${ }^{2,74,89,93,106}$ In a small subset of CCI rats, a protuberance similar to a leptomeningeal cyst formed several days after injury, and MRI showed findings similar to those in human infants with a leptomeningeal cyst. ${ }^{82}$ In P11 CCI, similar deformation of the lateral ventricle has been noted on histological analyses..$^{74}$ Additional histological and imaging studies are expected to correlate histological changes with MRI findings; however, those studies are beyond the scope of the present study. Initial findings with our P12 CCI model are consistent with results in previous preclinical models and imaging in human infants following impact TBI.

Detailed analyses of the DTI revealed chronic changes with a reduction in FA and an increase in $\mathrm{MD}, \mathrm{AD}$, and $\mathrm{RD}$ in bilateral white matter regions of CCI-veh brains, suggesting that the relatively focal CCI caused widespread chronic changes in diffusivity. Not surprisingly, the severity of the microstructural abnormalities was more prominent ipsilateral to the impact. These changes are similar to chronic white matter DTI abnormalities observed after TBI in children. ${ }^{18,95}$ To our knowledge no DTI studies of infant TBI have been performed, although these imaging studies have been used extensively to follow chronic outcomes in perinatal brain injury; ${ }^{33,58}$ therefore, DTI should be feasible to use in infants with TBI. The P12 CCI also caused a similar pattern of bilateral microstructural abnormalities in the cortex. Recently it was recognized that DTI can detect abnormalities in gray matter as well as in white matter. ${ }^{91}$ Results from the present study demonstrated the feasibility of using DTI as a detailed biomarker to quantify the severity and regional extent of injury and repair after infant TBI. Additional detailed regional analyses to clarify the extent of microstructural abnormalities throughout the brain using this model are underway.

Importantly, DTI abnormalities due to P12 CCI generally normalized with postinjury EPO treatment. These results suggest that DTI may be used as a biomarker to document responsiveness to emerging neuro-reparative strategies. In a preclinical model of toddler TBI with P17 CCI, a reduction in white matter RD related to an oral treatment regimen was observed. ${ }^{84}$ Indeed, detailed imaging studies have demonstrated differences in treatment groups when preterm infants were imaged at a term-equivalent age, 5,46 a relatively short interval of a few months to detect structural improvement in neurodevelopment in humans. These recent reports, ${ }^{5,46}$ in combination with data presented here, suggest that DTI may be used as an imaging biomarker in infant TBI to measure responsiveness to neuro-reparative strategies.

\section{Serum Biomarkers Reflecting Functional Outcomes After EPO Treatment}

Serum biomarkers will potentially allow stratification of patients and personalized tailoring of neuro-reparative strategies to the extent of injury, confounding comorbidities and individual tolerance for and response to injury. Accurate use of serum biomarkers requires an understanding of their biological basis in the CNS, which is probably complex in the developing brain..$^{37,39}$ In the present study we showed that cortical calpain activity is elevated 3 days after a CCI delivered on P12, as shown by increases in $\alpha$ IISDPs, KCC2-DPs, and GFAP-DPs. The observed elevation in $\alpha$ II-SDPs is consistent with findings from other TBI studies. ${ }^{8,70,86,107}$ While degradation of the cytoskeletal protein $\alpha$ II-S is a generic marker of neuronal injury, cerebral KCC2 levels more specifically reflect neuronal functional activity. In fact, $\mathrm{KCC} 2$ influences multiple components of neuronal activity including neuronal survival, inhibitory tone, synaptogenesis, and seizure threshold. $., 32,68,76,92,99$ Loss of KCC2 expression occurs with epilepsy, ${ }^{31}$ gliomas, ${ }^{15,65}$ trauma, ${ }^{11,12,67}$ and perinatal brain injury. ${ }^{37,36,79}$ Developmen- 
tally regulated molecules essential for cerebral function are vulnerable to calpain including KCC2, ${ }^{37,39,72,108}$ myelin basic protein, and phosphorylated neurofilament. ${ }^{39} \mathrm{~A}$ better understanding of the pattern of excess CNS calpain activity following infant TBI will guide the dosing of therapeutic interventions. These initial studies demonstrate that excess CNS calpain activity occurs following P12 CCI, as in other preclinical models of TBI. ${ }^{86}$

We focused on serum GFAP because it reflects both gliosis and calpain degradation, and GFAP-DPs are emerging as a leading TBI biomarker in humans. ${ }^{21,26,56,107}$ Using immunoblotting, we found elevations in total GFAP and GFAP-DPs in serum at 3 days after CCI, which corresponded to CNS levels. Huang and colleagues did not find elevated GFAP in serum at 24 hours after TBI; however, they used a different age (adults), injury mechanism (fluid percussion), and method of detection (enzyme-linked immunosorbent assay [ELISA] $),{ }^{30}$ differences that emphasize the need for age and assay specificity. Nonetheless, GFAP and GFAP-DPs and $\alpha$ II-SDPs are emerging as sensitive serum biomarkers. ${ }^{8,13,26}$ Clinically, elevated peripheral levels of GFAP following TBI are associated with unfavorable neurological outcomes. ${ }^{20,56}$ Notably, in children with TBI, GFAP outperforms other biomarkers such as S100 $\beta$ in detecting head trauma and predicting intracranial lesions on head CT. ${ }^{66}$ Moreover, GFAP levels are typically higher in serum following severe injury than after diffuse injury, and serum GFAP levels may be especially relevant in the pediatric patient population to detect contusion or intracerebral hemorrhage. ${ }^{105}$ Additionally, rapid measurement of GFAP-DPs may be useful in establishing or excluding the diagnosis of intracranial injury apparent on imaging across the spectrum of TBI. Thus, as an adjunct to current screening practices, measuring GFAP-DP levels may help avoid unnecessary CT scans without sacrificing sensitivity. ${ }^{56}$ While additional studies are needed to clarify the timing and regional extent of excess CNS calpain activity following P12 CCI, our initial results with serum GFAP suggest that calpain DPs will provide potentially useful serum biomarkers in infant TBI.

In the present study we also identified acute serum elevations in the proinflammatory cytokines TNF $\alpha$ and CXCL1 but not multiple other cytokines, which suggests that the increases in $\mathrm{TNF} \alpha$ and CXCL1 reflect activation of specific signaling mechanisms. In our P12 CCI model, TNF $\alpha$ elevations were transient, whereas CXCL1 levels remained elevated for 2-3 weeks after injury. To our knowledge this is the first report of elevated serum CXCL1 levels after infant TBI. As with other chemokines essential for neurodevelopment, CXCL1 signaling through its receptor CXCR2 regulates neuronal survival ${ }^{23}$ as well as oligodendrocyte precursor proliferation and migration. ${ }^{80,78,96,101}$ However, high levels of CXCL1 signaling are probably toxic to the developing brain. In preclinical models of chronic pain in the mature CNS, astrocytes secrete CXCL1, ${ }^{104}$ which stimulates $N$-methyl-D-aspartate (NMDA) receptors ${ }^{16}$ and probably calpain activation and KCC2 loss. ${ }^{37,108}$ We speculate that after TBI, astrocytes and/or microglia secrete excess CXCL1, probably driven by $\mathrm{TNF} \alpha{ }^{104}$ Indeed, CCI in Cxcr2-deficient mice induced less tissue and histological correlates of injury compared with wild-type mice, although functional outcomes were not altered ${ }^{88}$ Investigations of the mechanisms of TNF $\alpha$ and CXCL1 signaling are complex. Importantly, serum levels of CXCL1 that remained elevated for 2-3 weeks after P12 injury were attenuated by EPO treatment and collectively demonstrated the same pattern of improvement seen with functional studies and MRI biomarkers.

\section{Study Limitations}

This initial study has multiple limitations. The results presented here lead to several more questions including how P12 CCI alters the timing and location of CNS EPOR expression, gliosis from astrocytes and microglia, excess calpain activity, and inflammatory cytokines. These issues are being addressed with additional studies. A limitation in using young rodents is the challenge in performing a craniotomy without imparting injury to the underlying dura and brain. Even in adult mice, CCI produces changes in shamoperated rats compared with naïve animals. ${ }^{83}$ Another recent study of CCI in P14 rats also used a craniectomy. ${ }^{28}$ In that study anesthesia was induced and an incision without a craniectomy was performed in shams, while CCI rats underwent craniectomy with CCI. The CCI with craniectomy mimics the situation in infants who suffer severe TBI and are treated with decompressive craniectomy, a common clinical scenario. Because pilot studies showed that shams had some response to craniectomy and because our focus in the present study was to determine whether MRI and serum biomarkers could detect chronic injury changes, the experimental paradigm used here was most informative and consistent with the consensus that the presence or absence of craniectomy is especially important when function is evaluated at early time points. ${ }^{83}$ Subsequent studies with P12 rats to compare chronic injury in shams with craniectomy and CCI rats without craniectomy are underway. Finally, our studies were underpowered to determine any differences related to sex. Both sexes were used in all experiments to facilitate translation to humans. Our goal with this first report was to determine in infant impact TBI whether injury could be measured using MRI and serum biomarkers and the potential efficacy of EPO treatment.

\section{Conclusions}

In this initial study of CCI in P12 rats, we demonstrated that gait changes after CCI were accompanied by DTI abnormalities that resolved with EPO treatment. Similarly, chronic serum CXCL1 levels normalized with EPO treatment. We found that P12 rat CCI functional outcomes were reflected in MRI and serum biomarkers. Together, these results support both the use of MRI and serum biomarkers in infant TBI and the use of rats to test emerging neuro-reparative interventions.

\section{Acknowledgments}

We thank Eun-Hyoung Park, PhD, for her excellent assistance with the MATLAB illustrations. This project was supported in part by the Dedicated Health Research funds from the University of New Mexico School of Medicine and the Centers for Biomedical Research Excellence Pilot Award to Lauren L. Jantzie, PhD (CoBRE P30GM103400/PI:Liu), and Boston Children's Hospital. 


\section{References}

1. Adelson PD, Dixon CE, Robichaud P, Kochanek PM: Motor and cognitive functional deficits following diffuse traumatic brain injury in the immature rat. J Neurotrauma 14:99-108, 1997

2. Adelson PD, Fellows-Mayle W, Kochanek PM, Dixon CE: Morris water maze function and histologic characterization of two age-at-injury experimental models of controlled cortical impact in the immature rat. Childs Nerv Syst 29:4353, 2013

3. Adelson PD, Robichaud P, Hamilton RL, Kochanek PM: A model of diffuse traumatic brain injury in the immature rat. J Neurosurg 85:877-884, 1996

4. Aloizos S, Evodia E, Gourgiotis S, Isaia EC, Seretis C, Baltopoulos GJ: Neuroprotective effects of erythropoietin in patients with severe closed brain injury. Turk Neurosurg 25:552-558, 2015

5. Anblagan D, Bastin ME, Sparrow S, Piyasena C, Pataky $\mathrm{R}$, Moore EJ, et al: Tract shape modeling detects changes associated with preterm birth and neuroprotective treatment effects. Neuroimage Clin 8:51-58, 2015

6. Arango JI, Deibert CP, Brown D, Bell M, Dvorchik I, Adelson PD: Posttraumatic seizures in children with severe traumatic brain injury. Childs Nerv Syst 28:1925-1929, 2012

7. Aung WY, Mar S, Benzinger TL: Diffusion tensor MRI as a biomarker in axonal and myelin damage. Imaging Med 5:427-440, 2013

8. Berger RP, Hayes RL, Richichi R, Beers SR, Wang KK: Serum concentrations of ubiquitin C-terminal hydrolase-L1 and $\alpha$ II-spectrin breakdown product $145 \mathrm{kDa}$ correlate with outcome after pediatric TBI. J Neurotrauma 29:162-167, 2012

9. Blaesse P, Schmidt T: K-Cl cotransporter KCC2 - a moonlighting protein in excitatory and inhibitory synapse development and function. Pflugers Arch 467:615-624, 2015

10. Bondi CO, Semple BD, Noble-Haeusslein LJ, Osier ND, Carlson SW, Dixon CE, et al: Found in translation: understanding the biology and behavior of experimental traumatic brain injury. Neurosci Biobehav Rev [epub ahead of print], 2014

11. Bonislawski DP, Schwarzbach EP, Cohen AS: Brain injury impairs dentate gyrus inhibitory efficacy. Neurobiol Dis 25:163-169, 2007

12. Boulenguez P, Liabeuf S, Bos R, Bras H, Jean-Xavier C, Brocard C, et al: Down-regulation of the potassium-chloride cotransporter KCC2 contributes to spasticity after spinal cord injury. Nat Med 16:302-307, 2010

13. Boutté AM, Deng-Bryant Y, Johnson D, Tortella FC, Dave JR, Shear DA, et al: Serum glial fibrillary acidic protein predicts tissue glial fibrillary acidic protein break-down products and therapeutic efficacy after penetrating ballisticlike brain injury. J Neurotrauma [epub ahead of print], 2015

14. Brines M, Cerami A: Emerging biological roles for erythropoietin in the nervous system. Nat Rev Neurosci 6:484 494, 2005

15. Campbell SL, Robel S, Cuddapah VA, Robert S, Buckingham SC, Kahle KT, et al: GABAergic disinhibition and impaired KCC2 cotransporter activity underlie tumorassociated epilepsy. Glia 63:23-36, 2015

16. Cao DL, Zhang ZJ, Xie RG, Jiang BC, Ji RR, Gao YJ: Chemokine CXCL1 enhances inflammatory pain and increases NMDA receptor activity and COX-2 expression in spinal cord neurons via activation of CXCR2. Exp Neurol 261:328-336, 2014

17. Chen CY, Noble-Haeusslein LJ, Ferriero D, Semple BD: Traumatic injury to the immature frontal lobe: a new murine model of long-term motor impairment in the absence of psy- chosocial or cognitive deficits. Dev Neurosci 35:474-490, 2013

18. Dennis EL, Jin Y, Villalon-Reina JE, Zhan L, Kernan CL, Babikian T, et al: White matter disruption in moderate/ severe pediatric traumatic brain injury: advanced tract-based analyses. Neuroimage Clin 7:493-505, 2015

19. Dent KA, Christie KJ, Bye N, Basrai HS, Turbic A, Habgood M, et al: Oligodendrocyte birth and death following traumatic brain injury in adult mice. PLoS One 10:e0121541, 2015

20. Di Battista AP, Buonora JE, Rhind SG, Hutchison MG, Baker AJ, Rizoli SB, et al: Blood biomarkers in moderateto-severe traumatic brain injury: potential utility of a multimarker approach in characterizing outcome. Front Neurol 6: 110,2015

21. Diaz-Arrastia R, Wang KK, Papa L, Sorani MD, Yue JK, Puccio AM, et al: Acute biomarkers of traumatic brain injury: relationship between plasma levels of ubiquitin C-terminal hydrolase-L1 and glial fibrillary acidic protein. $\mathbf{J}$ Neurotrauma 31:19-25, 2014

22. Donelan JM, Shipman DW, Kram R, Kuo AD: Mechanical and metabolic requirements for active lateral stabilization in human walking. J Biomech 37:827-835, 2004

23. Edman LC, Mira H, Erices A, Malmersjö S, Andersson E, Uhlén P, et al: Alpha-chemokines regulate proliferation, neurogenesis, and dopaminergic differentiation of ventral midbrain precursors and neurospheres. Stem Cells 26:1891-1900, 2008

24. Fauchère JC, Dame C, Vonthein R, Koller B, Arri S, Wolf $\mathrm{M}$, et al: An approach to using recombinant erythropoietin for neuroprotection in very preterm infants. Pediatrics 122:375-382, 2008

25. Fauchère JC, Koller BM, Tschopp A, Dame C, Ruegger C, Bucher HU, et al: Safety of early high-dose recombinant erythropoietin for neuroprotection in very preterm infants. J Pediatr 167:52-57, 57.e1-57.e3, 2015

26. Fraser DD, Close TE, Rose KL, Ward R, Mehl M, Farrell $\mathrm{C}$, et al: Severe traumatic brain injury in children elevates glial fibrillary acidic protein in cerebrospinal fluid and serum. Pediatr Crit Care Med 12:319-324, 2011

27. Gobbel GT, Bonfield C, Carson-Walter EB, Adelson PD: Diffuse alterations in synaptic protein expression following focal traumatic brain injury in the immature rat. Childs Nerv Syst 23:1171-1179, 2007

28. Gu X, Wei ZZ, Espinera A, Lee JH, Ji X, Wei L, et al: Pharmacologically induced hypothermia attenuates traumatic brain injury in neonatal rats. Exp Neurol 267:135-142, 2015

29. Hellewell SC, Yan EB, Alwis DS, Bye N, MorgantiKossmann MC: Erythropoietin improves motor and cognitive deficit, axonal pathology, and neuroinflammation in a combined model of diffuse traumatic brain injury and hypoxia, in association with upregulation of the erythropoietin receptor. J Neuroinflammation 10:156, 2013

30. Huang XJ, Glushakova O, Mondello S, Van K, Hayes RL, Lyeth BG: Acute temporal profiles of serum levels of UCH-L1 and GFAP and relationships to neuronal and astroglial pathology following traumatic brain injury in rats. J Neurotrauma 32:1179-1189, 2015

31. Huberfeld G, Wittner L, Clemenceau S, Baulac M, Kaila $\mathrm{K}$, Miles R, et al: Perturbed chloride homeostasis and GABAergic signaling in human temporal lobe epilepsy. J Neurosci 27:9866-9873, 2007

32. Hübner CA, Stein V, Hermans-Borgmeyer I, Meyer T, Ballanyi K, Jentsch TJ: Disruption of KCC2 reveals an essential role of $\mathrm{K}-\mathrm{Cl}$ cotransport already in early synaptic inhibition. Neuron 30:515-524, 2001

33. Hüppi PS, Dubois J: Diffusion tensor imaging of brain development. Semin Fetal Neonatal Med 11:489-497, 2006 
34. Jantzie LL, Corbett CJ, Berglass J, Firl DJ, Flores J, Mannix R, et al: Complex pattern of interaction between in utero hypoxia-ischemia and intra-amniotic inflammation disrupts brain development and motor function. J Neuroinflammation 11:131, 2014

35. Jantzie LL, Corbett CJ, Firl DJ, Robinson S: Postnatal erythropoietin mitigates impaired cerebral cortical development following subplate loss from prenatal hypoxia-ischemia. Cereb Cortex 25:2683-2695, 2015

36. Jantzie LL, Getsy PM, Denson JL, Firl DJ, Maxwell JR, Rogers DA, et al: Prenatal hypoxia-ischemia induces abnormalities in CA3 microstructure, potassium chloride co-transporter 2 expression and inhibitory tone. Front Cell Neurosci 9:347, 2015

37. Jantzie LL, Getsy PM, Firl DJ, Wilson CG, Miller RH, Robinson S: Erythropoietin attenuates loss of potassium chloride co-transporters following prenatal brain injury. Mol Cell Neurosci 61:152-162, 2014

38. Jantzie LL, Miller RH, Robinson S: Erythropoietin signaling promotes oligodendrocyte development following prenatal systemic hypoxic-ischemic brain injury. Pediatr Res 74:658-667, 2013

39. Jantzie LL, Winer JL, Corbett CJ, Robinson S: Erythropoietin modulates cerebral and serum degradation products from excess calpain activation following prenatal hypoxia-ischemia. Dev Neurosci [epub ahead of print], 2015

40. Juul SE, McPherson RJ, Bauer LA, Ledbetter KJ, Gleason CA, Mayock DE: A phase I/II trial of high-dose erythropoietin in extremely low birth weight infants: pharmacokinetics and safety. Pediatrics 122:383-391, 2008

41. Keller M, Yang J, Griesmaier E, Gorna A, Sarkozy G, Urbanek M, et al: Erythropoietin is neuroprotective against NMDA-receptor-mediated excitotoxic brain injury in newborn mice. Neurobiol Dis 24:357-366, 2006

42. Kellert BA, McPherson RJ, Juul SE: A comparison of high-dose recombinant erythropoietin treatment regimens in brain-injured neonatal rats. Pediatr Res 61:451-455, 2007

43. Knabe W, Knerlich F, Washausen S, Kietzmann T, Sirén AL, Brunnett G, et al: Expression patterns of erythropoietin and its receptor in the developing midbrain. Anat Embryol (Berl) 207:503-512, 2004

44. Knabe W, Sirén AL, Ehrenreich H, Kuhn HJ: Expression patterns of erythropoietin and its receptor in the developing spinal cord and dorsal root ganglia. Anat Embryol (Berl) 210:209-219, 2005

45. Kocaman C, Yilmaz Y: Etiological analysis of presumed perinatal stroke. Brain Dev 34:133-139, 2012

46. Leuchter RH, Gui L, Poncet A, Hagmann C, Lodygensky GA, Martin E, et al: Association between early administration of high-dose erythropoietin in preterm infants and brain MRI abnormality at term-equivalent age. JAMA 312:817824,2014

47. Leventhal JM, Martin KD, Asnes AG: Fractures and traumatic brain injuries: abuse versus accidents in a US database of hospitalized children. Pediatrics 126:e104-e115, 2010

48. Mac Donald CL, Dikranian K, Bayly P, Holtzman D, Brody $\mathrm{D}$ : Diffusion tensor imaging reliably detects experimental traumatic axonal injury and indicates approximate time of injury. J Neurosci 27:11869-11876, 2007

49. Mannix R, Berglass J, Berkner J, Moleus P, Qiu J, Jantzie LL, et al: Sex differences in the effect of progesterone after controlled cortical impact in adolescent mice: a preliminary study. J Neurosurg 121:1337-1341, 2014

50. Mannix RC, Zhang J, Berglass J, Qui J, Whalen MJ: Beneficial effect of amyloid beta after controlled cortical impact. Brain Inj 27:743-748, 2013

51. Mannix RC, Zhang J, Park J, Lee C, Whalen MJ:
Detrimental effect of genetic inhibition of B-site APPcleaving enzyme 1 on functional outcome after controlled cortical impact in young adult mice. J Neurotrauma 28:1855-1861, 2011

52. Mannix RC, Zhang J, Park J, Zhang X, Bilal K, Walker K, et al: Age-dependent effect of apolipoprotein E4 on functional outcome after controlled cortical impact in mice. J Cereb Blood Flow Metab 31:351-361, 2011

53. Mazur M, Miller RH, Robinson S: Postnatal erythropoietin treatment mitigates neural cell loss after systemic prenatal hypoxic-ischemic injury. J Neurosurg Pediatr 6:206-221, 2010

54. McAdams RM, McPherson RJ, Mayock DE, Juul SE: Outcomes of extremely low birth weight infants given early high-dose erythropoietin. J Perinatol 33:226-230, 2013

55. McKinlay A, Grace RC, Horwood LJ, Fergusson DM, Ridder EM, MacFarlane MR: Prevalence of traumatic brain injury among children, adolescents and young adults: prospective evidence from a birth cohort. Brain Inj 22:175181,2008

56. McMahon PJ, Panczykowski DM, Yue JK, Puccio AM, Inoue T, Sorani MD, et al: Measurement of the glial fibrillary acidic protein and its breakdown products GFAP-BDP biomarker for the detection of traumatic brain injury compared to computed tomography and magnetic resonance imaging. J Neurotrauma 32:527-533, 2015

57. Meng Y, Xiong Y, Mahmood A, Zhang Y, Qu C, Chopp M: Dose-dependent neurorestorative effects of delayed treatment of traumatic brain injury with recombinant human erythropoietin in rats. J Neurosurg 115:550-560, 2011

58. Ment LR, Hirtz D, Hüppi PS: Imaging biomarkers of outcome in the developing preterm brain. Lancet Neurol 8:1042-1055, 2009

59. Miller TR, Steinbeigle R, Wicks A, Lawrence BA, Barr M, Barr RG: Disability-adjusted life-year burden of abusive head trauma at ages 0-4. Pediatrics 134:e1545-e1550, 2014

60. Miskowiak KW, Ehrenreich H, Christensen EM, Kessing LV, Vinberg M: Recombinant human erythropoietin to target cognitive dysfunction in bipolar disorder: a doubleblind, randomized, placebo-controlled phase 2 trial. J Clin Psychiatry 75:1347-1355, 2014

61. Miskowiak KW, Vinberg M, Christensen EM, Bukh JD, Harmer CJ, Ehrenreich H, et al: Recombinant human erythropoietin for treating treatment-resistant depression: a double-blind, randomized, placebo-controlled phase 2 trial. Neuropsychopharmacology 39:1399-1408, 2014

62. Murray KJ, Griffin R, Rue LW III, McGwin G Jr: Recent trends in television tip over-related injuries among children aged 0-9 years. Inj Prev 15:240-243, 2009

63. Ohls RK, Kamath-Rayne BD, Christensen RD, Wiedmeier SE, Rosenberg A, Fuller J, et al: Cognitive outcomes of preterm infants randomized to darbepoetin, erythropoietin, or placebo. Pediatrics 133: 1023-1030, 2014

64. O'Neill BR, Handler MH, Tong S, Chapman KE: Incidence of seizures on continuous EEG monitoring following traumatic brain injury in children. J Neurosurg Pediatr 16:167-176, 2015

65. Pallud J, Le Van Quyen M, Bielle F, Pellegrino C, Varlet P, Labussiere M, et al: Cortical GABAergic excitation contributes to epileptic activities around human glioma. Sci Transl Med 6:244ra89, 2014

66. Papa L, Ramia MM, Edwards D, Johnson BD, Slobounov SM: Systematic review of clinical studies examining biomarkers of brain injury in athletes after sports-related concussion. J Neurotrauma 32:661-673, 2015

67. Payne JA, Rivera C, Voipio J, Kaila K: Cation-chloride cotransporters in neuronal communication, development and trauma. Trends Neurosci 26:199-206, 2003 
68. Pellegrino C, Gubkina O, Schaefer M, Becq H, Ludwig A, Mukhtarov M, et al: Knocking down of the KCC2 in rat hippocampal neurons increases intracellular chloride concentration and compromises neuronal survival. J Physiol 589:2475-2496, 2011

69. Peng W, Xing Z, Yang J, Wang Y, Wang W, Huang W: The efficacy of erythropoietin in treating experimental traumatic brain injury: a systematic review of controlled trials in animal models. J Neurosurg 121:653-664, 2014

70. Pike BR, Zhao X, Newcomb JK, Posmantur RM, Wang KK, Hayes RL: Regional calpain and caspase-3 proteolysis of alpha-spectrin after traumatic brain injury. Neuroreport 9:2437-2442, 1998

71. Ponce LL, Navarro JC, Ahmed O, Robertson CS: Erythropoietin neuroprotection with traumatic brain injury. Pathophysiology 20:31-38, 2013

72. Puskarjov M, Ahmad F, Kaila K, Blaesse P: Activitydependent cleavage of the $\mathrm{K}-\mathrm{Cl}$ cotransporter $\mathrm{KCC} 2$ mediated by calcium-activated protease calpain. J Neurosci 32:11356-11364, 2012

73. Quayle KS, Powell EC, Mahajan P, Hoyle JD Jr, Nadel FM, Badawy MK, et al: Epidemiology of blunt head trauma in children in U.S. emergency departments. N Engl J Med 371:1945-1947, 2014

74. Raghupathi R, Huh JW: Diffuse brain injury in the immature rat: evidence for an age-at-injury effect on cognitive function and histopathologic damage. J Neurotrauma 24:1596-1608, 2007

75. Rivara FP, Vavilala MS, Durbin D, Temkin N, Wang J, O'Connor SS, et al: Persistence of disability 24 to 36 months after pediatric traumatic brain injury: a cohort study. J Neurotrauma 29:2499-2504, 2012

76. Rivera C, Voipio J, Payne JA, Ruusuvuori E, Lahtinen H, Lamsa K, et al: The $\mathrm{K}^{+} / \mathrm{Cl}^{-}$co-transporter $\mathrm{KCC} 2$ renders GABA hyperpolarizing during neuronal maturation. Nature 397:251-255, 1999

77. Robertson CS, Hannay HJ, Yamal JM, Gopinath S, Goodman JC, Tilley BC, et al: Effect of erythropoietin and transfusion threshold on neurological recovery after traumatic brain injury: a randomized clinical trial. JAMA 312:36-47, 2014

78. Robinson S, Franic LA: Chemokine GRO1 and the spatial and temporal regulation of oligodendrocyte precursor proliferation. Dev Neurosci 23:338-345, 2001

79. Robinson S, Mikolaenko I, Thompson I, Cohen ML, Goyal M: Loss of cation-chloride cotransporter expression in preterm infants with white matter lesions: implications for the pathogenesis of epilepsy. J Neuropathol Exp Neurol 69:565-572, 2010

80. Robinson S, Tani M, Strieter RM, Ransohoff RM, Miller $\mathrm{RH}$ : The chemokine growth-regulated oncogene-alpha promotes spinal cord oligodendrocyte precursor proliferation. J Neurosci 18:10457-10463, 1998

81. Rutkoski JD, Sippey M, Gaines BA: Traumatic television tip-overs in the pediatric patient population. J Surg Res 166:199-204, 2011

82. Sanford RA: Prevention of growing skull fractures: report of 2 cases. J Neurosurg Pediatr 5:213-218, 2010

83. Sashindranath M, Daglas M, Medcalf RL: Evaluation of gait impairment in mice subjected to craniotomy and traumatic brain injury. Behav Brain Res 286:33-38, 2015

84. Schober ME, Requena DF, Abdullah OM, Casper TC, Beachy J, Malleske D, et al: Dietary docosahexaenoic acid improves cognitive function, tissue sparing, and magnetic resonance imaging indices of edema and white matter injury in the immature rat after traumatic brain injury. $\mathbf{J}$ Neurotrauma [epub ahead of print], 2015

85. Schober ME, Requena DF, Block B, Davis LJ, Rodesch C, Casper TC, et al: Erythropoietin improved cognitive func- tion and decreased hippocampal caspase activity in rat pups after traumatic brain injury. J Neurotrauma 31:358-369, 2014

86. Schober ME, Requena DF, Davis LJ, Metzger RR, Bennett KS, Morita D, et al: Alpha II Spectrin breakdown products in immature Sprague Dawley rat hippocampus and cortex after traumatic brain injury. Brain Res 1574:105-112, 2014

87. Semple BD, Blomgren K, Gimlin K, Ferriero DM, NobleHaeusslein LJ: Brain development in rodents and humans: Identifying benchmarks of maturation and vulnerability to injury across species. Prog Neurobiol 106-107:1-16, 2013

88. Semple BD, Bye N, Ziebell JM, Morganti-Kossmann MC: Deficiency of the chemokine receptor CXCR2 attenuates neutrophil infiltration and cortical damage following closed head injury. Neurobiol Dis 40:394-403, 2010

89. Semple BD, Noble-Haeusslein LJ, Jun Kwon Y, Sam PN, Gibson AM, Grissom S, et al: Sociosexual and communication deficits after traumatic injury to the developing murine brain. PLoS One 9:e103386, 2014

90. Shang Y, Wu Y, Yao S, Wang X, Feng D, Yang W: Protective effect of erythropoietin against ketamine-induced apoptosis in cultured rat cortical neurons: involvement of PI3K/Akt and GSK-3 beta pathway. Apoptosis 12:21872195, 2007

91. Sierra A, Laitinen T, Gröhn O, Pitkänen A: Diffusion tensor imaging of hippocampal network plasticity. Brain Struct Funct 220:781-801, 2015

92. Sivakumaran S, Cardarelli RA, Maguire J, Kelley MR, Silayeva L, Morrow DH, et al: Selective inhibition of KCC2 leads to hyperexcitability and epileptiform discharges in hippocampal slices and in vivo. J Neurosci 35:8291-8296, 2015

93. Soblosky JS, Matthews MA, Davidson JF, Tabor SL, Carey ME: Traumatic brain injury of the forelimb and hindlimb sensorimotor areas in the rat: physiological, histological and behavioral correlates. Behav Brain Res 79:79-92, 1996

94. Statler PA, McPherson RJ, Bauer LA, Kellert BA, Juul SE: Pharmacokinetics of high-dose recombinant erythropoietin in plasma and brain of neonatal rats. Pediatr Res 61:671675, 2007

95. Tasker RC, Westland AG, White DK, Williams GB: Corpus callosum and inferior forebrain white matter microstructure are related to functional outcome from raised intracranial pressure in child traumatic brain injury. Dev Neurosci 32:374-384, 2010

96. Tsai HH, Frost E, To V, Robinson S, ffrench-Constant C, Geertman R, et al: The chemokine receptor CXCR2 controls positioning of oligodendrocyte precursors in developing spinal cord by arresting their migration. Cell 110:373-383, 2002

97. Tsai PT, Ohab JJ, Kertesz N, Groszer M, Matter C, Gao J, et al: A critical role of erythropoietin receptor in neurogenesis and post-stroke recovery. J Neurosci 26:1269-1274, 2006

98. Webster SJ, Van Eldik LJ, Watterson DM, Bachstetter AD: Closed head injury in an age-related Alzheimer mouse model leads to an altered neuroinflammatory response and persistent cognitive impairment. J Neurosci 35:6554-6569, 2015

99. Winkelmann A, Semtner M, Meier JC: Chloride transporter KCC2-dependent neuroprotection depends on the N-terminal protein domain. Cell Death Dis 6:e1776, 2015

100. Wu Q, Miller RH, Ransohoff RM, Robinson S, Bu J, Nishiyama A: Elevated levels of the chemokine GRO-1 correlate with elevated oligodendrocyte progenitor proliferation in the jimpy mutant. J Neurosci 20:2609-2617, 2000

101. Wu YW, Bauer LA, Ballard RA, Ferriero DM, Glidden DV, Mayock DE, et al: Erythropoietin for neuroprotection in neonatal encephalopathy: safety and pharmacokinetics. Pediatrics 130:683-691, 2012 
102. Xiong Y, Mahmood A, Meng Y, Zhang Y, Qu C, Schallert $\mathrm{T}$, et al: Delayed administration of erythropoietin reducing hippocampal cell loss, enhancing angiogenesis and neurogenesis, and improving functional outcome following traumatic brain injury in rats: comparison of treatment with single and triple dose. J Neurosurg 113:598-608, 2010

103. Xiong Y, Mahmood A, Zhang Y, Meng Y, Zhang ZG, Qu C, et al: Effects of posttraumatic carbamylated erythropoietin therapy on reducing lesion volume and hippocampal cell loss, enhancing angiogenesis and neurogenesis, and improving functional outcome in rats following traumatic brain injury. J Neurosurg 114:549-559, 2011

104. Xu J, Zhu MD, Zhang X, Tian H, Zhang JH, Wu XB, et al: $\mathrm{NF \kappa B}$-mediated CXCL1 production in spinal cord astrocytes contributes to the maintenance of bone cancer pain in mice. J Neuroinflammation 11:38, 2014

105. Yokobori S, Hosein K, Burks S, Sharma I, Gajavelli S, Bullock R: Biomarkers for the clinical differential diagnosis in traumatic brain injury-a systematic review. CNS Neurosci Ther 19:556-565, 2013

106. Zhang Z, Saraswati M, Koehler RC, Robertson C, Kannan $\mathrm{S}$ : A new rabbit model of pediatric traumatic brain injury. $\mathbf{J}$ Neurotrauma 32:1369-1379, 2015

107. Zhang Z, Zoltewicz JS, Mondello S, Newsom KJ, Yang Z, Yang B, et al: Human traumatic brain injury induces autoantibody response against glial fibrillary acidic protein and its breakdown products. PLoS One 9:e92698, 2014

108. Zhou HY, Chen SR, Byun HS, Chen H, Li L, Han HD, et al: $N$-methyl-D-aspartate receptor- and calpain-mediated proteolytic cleavage of $\mathrm{K}^{+}-\mathrm{Cl}^{-}$cotransporter-2 impairs spinal chloride homeostasis in neuropathic pain. J Biol Chem 287:33853-33864, 2012

\section{Disclosures}

Dr. Meehan receives royalties from ABC-Clio Publishing and
Wolters Kluwer. He is under contract with ABC-Clio Publishing and Springer International Publishing for 1 future book each. His research is funded in part by a grant from the National Football League Players Association and by philanthropic support from the National Hockey League Alumni Association through the Corey C. Griffin Pro-Am Tournament.

\section{Author Contributions}

Conception and design: Robinson, Winer, Sillerud, Tasker, Jantzie. Acquisition of data: Winer, Berkner, Chan, Denson, Maxwell, Yang, Sillerud, Jantzie. Analysis and interpretation of data: Robinson, Winer, Chan, Denson, Maxwell, Yang, Sillerud, Tasker, Meehan, Mannix, Jantzie. Drafting the article: Robinson, Jantzie. Critically revising the article: all authors. Reviewed submitted version of manuscript: all authors. Approved the final version of the manuscript on behalf of all authors: Robinson, Jantzie. Statistical analysis: Robinson, Sillerud, Tasker, Jantzie. Administrative/ technical/material support: Jantzie. Study supervision: Robinson, Mannix, Jantzie.

\section{Supplemental Information}

\section{Previous Presentations}

Portions of these results were presented as abstracts at the 5th Penn State Hershey International Conference on Pediatric Abusive Head Trauma in Park City, Utah, in July 2015 (oral), and the Congress of Neurological Surgeons in New Orleans, Louisiana, in September 2015 (poster).

\section{Correspondence}

Shenandoah Robinson, Department of Neurosurgery, Boston Children's Hospital, 300 Longwood Ave., Boston, MA 02115. email: shenandoah.robinson@childrens.harvard.edu. 